\title{
COMPROMISO ORGANIZACIONAL DE LOS TRABAJADORES DE UNA UNIVERSIDAD PÚBLICA DE LIMA Y SU RELACIÓN CON ALGUNAS VARIABLES DEMÓGRAFICAS
}

\author{
Alejandro E. Loli Pineda ${ }^{*}$
}

\begin{abstract}
RESUMEN
La presente investigación trata de conocer el compromiso organizacional de los trabajadores no docentes de una universidad pública de Lima y su relación con algunas variables demográficas en una muestra de 205 personas de un total de 20 Facultades. Los resultados arrojan una tendencia favorable de los trabajadores hacia el compromiso organizacional y el análisis de los factores nos indica correlación muy significativa y positiva entre satisfacción general con el trabajo y compromiso con el trabajo, compromiso afectivo, jerarquía del puesto y sentimiento de permanencia; mientras que, la relación entre el primero y el compromiso de conveniencia es muy significativa pero negativa. Igual ocurre entre el compromiso con el trabajo y compromiso de conveniencia. También encontramos asociación muy significativa y positiva entre el compromiso con el trabajo y el compromiso afectivo, sentimiento de permanencia y jerarquía del puesto.
\end{abstract}

Entre tanto, las variables demográficas respecto al compromiso organizacional no arroja ninguna relación; pero un análisis separado por cada uno de los factores del compromiso organizacional permite encontrar relación importante con educación, antigüedad en la universidad, antigüedad en el puesto actual y puesto actual. Las demás variables demográficas como sexo, edad, condiciones laborales, estado civil y número de trabajadores, si bien muestran tendencias de compromiso, en algunos casos no son variables estadísticamente diferenciadoras.

Palabras clave: Compromiso, organización, trabajadores, componentes del compromiso, variables demográficas.

\begin{abstract}
The present investigation tries to know the organizational commitment about the workers not teachers of a public university of Lima and its relation with some demographic variables in a sample of 205 people of a whole of 20 faculties. The results throw a suitable trend of the workers towards the organizational commitment and the analysis of the factors indicates very significant and positive correlation between general satisfaction with the work and
\end{abstract}

* Docente Principal. Facultad de Psicología de la UNMSM.

E-mail: alolip@unmsm.edu.pe 
work commitment, affective commitment, hierarchy of the position and permanency feeling; whereas, the relation between the first one and the commitment of convenience is very significant but negative. The same happens between the commitment with the work and commitment of convenience. Also we find very significant and positive association between the commitment with the work and the affective commitment, permanency feeling and hierarchy of the position.

In the mean while, the demographic variables with regard to the organizational commitment throws no relation; but separated analysis for each one of the organizational commitment factors allows to find important relation with education, antiquity in the university, antiquity in the current position and current position. Other demographic variables such as sex, age, labour conditions, marital status and workers' number, though they show trends of commitment in some cases are not changeable statistically variables.

key words: Commitment, organization, workers, components of the commitment, demographic variables.

\section{INTRODUCCIÓN}

Una preocupación permanente de los directivos de las organizaciones ha sido siempre cómo satisfacer a sus trabajadores para lograr un mejor rendimiento o una mayor productividad. La probabilidad que aún exista directivos que sigan pensando así es muy alta, especialmente en nuestros países latinoamericanos, donde la investigación es escasa y difícil de emprender por diversas razones; aunque en los últimos tiempos, los problemas financieros, la falta de apoyo institucional, la resistencia en invertir en la formación de investigadores, entre otros, está cambiando. Mientras que en los países desarrollados se ha invertido ingentes cantidades de dinero desde que se realizaron las primeras investigaciones sobre satisfacción laboral, allá por la década de los años 40 del siglo pasado. Sin embargo, una revisión detallada de la literatura nos dice que es difícil encontrar una investigación suficiente, necesaria y clara para afirmar que un trabajador satisfecho sea altamente productivo, por el contrario, en la práctica es posible encontrar trabajadores satisfechos y poco productivos.

Así, para los investigadores de las organizaciones, cómo elevar el rendimiento y la productividad del capital humano, sigue siendo un reto; mientras que para los directivos contar con colaboradores con mística, espíritu de cuerpo o con la «camiseta bien puesta» (teniendo como mira el mejoramiento de la productividad), más que una aspiración, es una meta que se debe lograr. Es decir, directivos y trabajadores que entiendan que la única manera de alcanzar el progreso y el bienestar individual y de la organización es elevando el rendimiento y la productividad, y ello requiere de trabajadores comprometidos con su organización (Gibson, Ivancebich,y Donnelly, 1994).

En ese camino, estudiosos del comportamiento humano vienen trabajando para conocer cuáles son esos vínculos que los compromete a las personas para mantenerse más cerca, identificados o involucrados a su organización (Dessler, 1996). El presente trabajo forma parte de esta preocupación.

Evidentemente, estamos frente a un componente intrínseco tanto para el sujeto que se incorpora a trabajar como para la organización que lo recibe. Se trata de un contrato que va más allá de lo formal y legal, al que se le denomina contrato psicológico. Mientras una 
persona puede estar obligada formalmente a desempeñar una labor, no necesariamente se liga afectivamente a la organización (Arias Galicia, 1972) El contrato psicológico implica la existencia de un conjunto de expectativas recíprocas y están implícitas dentro de la interacción trabajador-organización.

De hecho, el compromiso de los trabajadores con la organización constituye una parte trascendente en la efectividad y competitividad de la organización. Meyer y Allen (1991) definen el compromiso organizacional como un «... estado psicológico que caracteriza la relación entre una persona y una organización», donde las expectativas de ambas partes son muy propias, individuales, diferentes y amplias que casi nunca es posible expresar todos de aspectos en una relación formal (incluso legal). Mathieu y Zajac (1990), después de revisar los escritos de un conjunto de investigadores llegaron a señalar que las definiciones sobre compromiso tienen en común expresiones como «... vínculo o lazo del individuo con la organización» (Bayona, C. y otros, 2000).

Existen otras definiciones últimas sobre compromiso organizacional y todas ellas mantienen el sentido del vínculo y la afectividad como característica básica: «...apego afectivo» (Bayona, C.,Goñi, S. y Madorrán, C., 2000); «...estado interno de la persona» (Lagomarsino, R., 2003), «...vínculos que mantienen a un sujeto apegado a una empresa (Arciniega, L. s/ f), «...deber moral o psicológico adquirido hacia una persona, una situación o una institución (Arias Galicia, F. 2003), entre otros.

Así, Steers (1977) definió el compromiso como «... la fuerza relativa de identificación y de involucramiento de un individuo con una organización». Tomando como modelo tres fuentes del compromiso: Características personales, características del trabajo y experiencias en el trabajo; se puso en prueba el modelo y se encontró que las organizaciones pueden influir más fácilmente sobre las dos últimas fuentes, en cambio transformar la personalidad sería mucho más difícil y cuestionable desde un ángulo ético.

Entre tanto, Meyer y Allen (1991), tras definir el compromiso (señalado antes), indicaron que dicha relación entre una persona y una organización, presenta consecuencias respecto a la decisión para continuar en la organización o dejarla. Estos autores propusieron tres componentes del compromiso: Afectivo (lazos emocionales que las personas forjan con la organización al satisfacer sus necesidades y expectativas), de continuidad o conveniencia (conciencia de la persona respecto a la inversión en tiempo y esfuerzo que se perderían en caso de dejar la organización para encontrar otro empleo) y normativo (la creencia en la lealtad a la organización en correspondencia a ciertas prestaciones).

Entre tanto, existen otros autores que plantean propuestas que difieren en los términos, pero que coinciden en su contenido. Así encontramos la propuesta de Lagomarsino, R. (2003): compromiso económico, de crecimiento y moral; lo presentado por Bayona, C. y otras (2000): compromiso actitudinal, el calculativo y el normativo, orientado en el mismo sentido de la propuesta de Mathieu y Zajac (1990): compromiso actitudinal y calculador; o el compromiso de identificación, de membresía y de lealtad de Claure Rocha, M. y Böhrt,M. (2003), o compromisos múltiples de Reichers (1985, en Varona, F. 1993). Excepto esta última propuesta, todas son similares a lo planteado por Meyer y Allen (1991).

Por su lado, las investigaciones han demostrado que existe relación entre las dimensiones propuestas por Meyer y Allen (1991), probablemente la dimensión que mayor controversia ha suscitado es el compromiso afectivo y continuo (Antón, C. y Gonzáles, L., 2005), cuya 
relación es moderada. Un estudio sobre el compromiso organizacional como herramienta de gestión de recursos humanos, en una muestra de 138 docentes de la Universidad de Navarra, España, concluye que no existe correlación entre compromiso actitudinal y compromiso de continuidad (nótese que el compromiso actitudinal como el de continuidad se conceptualizan en los términos de Meyer y Allen*); que las variables relacionadas con el trabajo y el grupo son las que presentan valores más altos con el compromiso actitudinal, así como la edad y no otra variable resulta significativa en este estudio (Bayona, C., 2000).

Otro trabajo desarrollado sobre compromiso personal hacia la organización y la intención de permanencia, en una muestra de 177 empleados de una institución privada de beneficencia, arrojó que el factor más importante para lograr el compromiso e incrementar la intención de permanencia es el apoyo organizacional, siendo éste preponderante sobre el compromiso afectivo, y éste último a su vez ejerce mayor peso sobre la satisfacción general en el trabajo de los empleados; finalmente, la expectativa de recompensa quedó en segundo lugar en cuanto a la influencia sobre el compromiso normativo (Arias Galicia, F., 2001).

Claure Rocha, M. y Börht Pelaez, M. (2003), también investigaron sobre tres dimensiones del compromiso organizacional: identificación, membresía y lealtad, en una muestra de 26 sujetos $(16.25 \%)$ de un total de 160 trabajadores de una empresa privada de servicios, concluyendo que el componente identificación tiene una relación significativa con la variable compromiso organizacional, que el componente membresía tiende a ser débil y poco desarrollado en el grupo estudiado, y que el componente lealtad es más alto en el grupo de 3 a 6 años de servicio en la organización. Así mismo, se encontró correlación significativa entre antigüedad e identificación, entre antigüedad y lealtad, y entre antigüedad y compromiso organizacional.

En el Perú destaca la investigación desarrollada por Arias, F.; Valega, D.; Loli, A. y Quintana, M. (2003) sobre compromiso organizacional y su relación con algunos factores demográficos, en una muestra de 190 sujetos de diversas organizaciones privadas y públicas y de diferentes tamaños. Los autores encontraron una correlación alta entre compromiso afectivo y satisfacción general con el trabajo, también entre compromiso afectivo y compromiso con el trabajo, igual que compromiso afectivo y claridad del rol, en cambio no fue significativa la relación con jerarquía del puesto. Por otro lado, las correlaciones entre el compromiso de conveniencia y las demás variables resultaron inferiores, siendo la más alta con claridad del rol, seguida por satisfacción con el trabajo. En cambio ninguna de las variables demográficas denotó asociación importante con el compromiso de conveniencia.

En concordancia con las perspectivas de las investigaciones señaladas, muchas instituciones que han comprendido que no pueden seguir viviendo sólo de un pasado exitoso -como es el caso de la presente investigación- se están involucrando en una carrera hacia la calidad en la gestión y, siendo el compromiso de las personas con la organización una forma de lograr efectividad en las organizaciones para el logro de la competitividad y el éxito, conocer el compromiso de sus recursos humanos, es más que un reto, una necesidad apremiante en el momento actual.

\section{Hipótesis}

Existe compromiso organizacional de los trabajadores no docentes con su centro de trabajo y éste está relacionado con algunos factores demográficos. 


\section{MÉTODOS}

\section{Muestra}

La muestra estuvo constituida por 205 trabajadores no docentes de una universidad pública de Lima (pertenecen a 20 facultades que forman parte de la organización). Estos participantes representan aproximadamente el $10 \%$ de la población total y fueron seleccionados al azar. Excepto la condición laboral que fue proporcional al número total de trabajadores por Facultad, como es el caso de Medicina Humana, donde participaron 14 trabajadores a razón de 7 nombrados y 7 contratados y en Ing. Electrónica y Mecánica de Fluidos 5 nombrados y seis contratados por existir mayor número de contratados.

\section{Composicion de la muestra según variables demograficas}

\begin{tabular}{|c|c|c|c|c|c|c|c|c|c|c|}
\hline COND. LABORAL & \multicolumn{4}{|c|}{ Grupo etario } & \multicolumn{2}{|c|}{ SEXO } & \multicolumn{3}{|c|}{ ESTADO CIVIL } & EDUCACIÓN \\
\hline Nombrado contratado & $21-30$ & $31-40$ & $41-50$ & $51+$ & M & $\mathrm{F}$ & S. C. I & D. & & S. T. SI. SC. Pr PG \\
\hline 102 & 54 & 64 & 58 & 29 & 80 & 125 & 77100 & 8 & 13 & $\begin{array}{llllll}29 & 65 & 40 & 43 & 22 & 6\end{array}$ \\
\hline 205 & \multicolumn{4}{|c|}{205} & \multicolumn{2}{|c|}{205} & \multicolumn{3}{|c|}{205} & 205 \\
\hline
\end{tabular}

\section{Instrumento}

El estudio se llevó a cabo con un cuestionario adaptado sobre la base del instrumento preparado por Fernando Arias Galicia, cuyo contenido está conformado por reactivos relativos a Compromiso afectivo, de conveniencia o continuidad y sentimiento de permanencia o normativo (Meyer y Allen,1991); Satisfacción General en el Trabajo (Eisenberger,1997), referido a respuestas afectivas dadas en torno su centro de trabajo; Claridad del Rol (Brown y Leigh, 1996), referido a la diafanidad de las expectativas así como a las normas de trabajo consistentes; Compromiso con el Trabajo (Lodahl y Kejner, 1965), referido al grado en el cual la persona ha acogido los valores respecto a la bondad de sus labores y la importancia de las mismas para su propia valía.

\section{a) Validez factorial}

Aplicado el instrumento y sometido a Análisis Factorial exploratorio (Rotación Varimax con Kaise) nos permitió confirmar los factores teóricos e identificar otros factores, probablemente debido a las influencias de nuestro contexto sociocultural. Los componentes señalados antes en el cuestionario trabajado por Fernando Arias Galicia (2001) difieren muy sutilmente de los factores extraidos, lo que permite dar fe de su validez confirmatorio.

Así, la variable Claridad del rol apareció difusa y se procedió a no tomar en cuenta dichos ítemes, surgiendo en cambio un nuevo factor al que denominamos Sentimiento de Permanencia (sin duda, equivalente a compromiso normativo), referido a permanecer horas adicionales diarias o semanales y sentirse bien por ello, tal vez por el sentido del deber o en compensación con las prestaciones recibidas, otros dirían por lealtad, en el sentido de cumplimiento y respeto a la organización (Claure, M., 2003). Igualmente se eliminaron algunos ítemes de Satisfacción general en el trabajo y otros. En el primer caso, porque el peso factorial era inferior a 0.46 y los predictores de dicho factor eran proporcionalmente excesivos en relación a los demás; en los otros casos, porque no alcanzaron un peso superior a 0.20 . 


\begin{tabular}{|c|c|c|c|c|c|c|}
\hline \multirow[b]{2}{*}{ ÍTEMES } & \multicolumn{6}{|c|}{ FACTORES DE COMPROMISO ORGANIZACIONAL } \\
\hline & $\begin{array}{c}\text { Satisfacción } \\
\text { General en } \\
\text { Trabajo } \\
\end{array}$ & $\begin{array}{c}\text { Compromiso } \\
\text { con el Trabajo }\end{array}$ & $\begin{array}{l}\text { Compromiso de } \\
\text { Conveniencia }\end{array}$ & $\begin{array}{c}\text { Compromiso } \\
\text { Afectivo }\end{array}$ & $\begin{array}{c}\text { Sentimiento } \\
\text { de Permanencia }\end{array}$ & $\begin{array}{l}\text { Jerarquía } \\
\text { del Puesto }\end{array}$ \\
\hline $\begin{array}{l}\text { ST } 102 \\
\text { ST } 76\end{array}$ & .77 & \multirow{47}{*}{$\begin{array}{l}.68 \\
.63 \\
.59 \\
.53 \\
.52 \\
.49 \\
.49 \\
.47 \\
.43 \\
.41 \\
.40 \\
.38 \\
.37 \\
.35 \\
.34\end{array}$} & & & & \\
\hline ST 96 & .75 & & & & & \\
\hline $\begin{array}{l}\text { ST } 100 \\
\text { ST 92 }\end{array}$ & .73 & & & & & \\
\hline ST 60 & .69 & & & & & \\
\hline $\begin{array}{l}\text { ST 88 } \\
\text { ST 35 }\end{array}$ & $\begin{array}{l}.69 \\
.68\end{array}$ & & & & & \\
\hline $\begin{array}{l}\text { ST 85 } \\
\text { ST 62 }\end{array}$ & $\begin{array}{l}.68 \\
.67\end{array}$ & & & & & \\
\hline ST 94 & $\begin{array}{l}66 \\
64\end{array}$ & & & & & \\
\hline $\begin{array}{l}\text { TT 66 } \\
\text { ST 50 }\end{array}$ & $\begin{array}{l}.64 \\
.64 \\
6.54\end{array}$ & & & & & \\
\hline $\begin{array}{l}\text { ST 95 } \\
\text { ST 36 }\end{array}$ & .63 & & & & & \\
\hline $\begin{array}{l}\text { ST 55 } \\
\text { ST } 101\end{array}$ & $\begin{array}{l}.60 \\
.58\end{array}$ & & & & & \\
\hline $\begin{array}{l}\text { ST } 63 \\
\text { ST 44 }\end{array}$ & .57 & & & & & \\
\hline ST 11 & .55 & & & & & \\
\hline $\begin{array}{l}\text { ST 74 } \\
\text { ST 98 }\end{array}$ & $\begin{array}{l}.51 \\
.48\end{array}$ & & & & & \\
\hline $\begin{array}{l}\text { ST } 104 \\
\text { ST 89 }\end{array}$ & $\begin{array}{l}.48 \\
.46\end{array}$ & & & & & \\
\hline $\begin{array}{l}\text { С } 23 \\
\text { СТ } 29\end{array}$ & & & & & & \\
\hline $\begin{array}{lll}\text { CT } 27 \\
\text { CT } 8\end{array}$ & & & & & & \\
\hline $\begin{array}{l}\text { CT15 } \\
\text { CT } 40\end{array}$ & & & & & & \\
\hline CT 22 & & & & & & \\
\hline $\begin{array}{l}\text { CT } 7 \\
\text { СТ } 99\end{array}$ & & & & & & \\
\hline $\begin{array}{l}\text { CT } 54 \\
\text { CT } 105\end{array}$ & & & & & & \\
\hline CT 16 & & & & & & \\
\hline CT 81 & & & & & & \\
\hline $\begin{array}{l}\text { CT 77 } \\
\text { CT } 10\end{array}$ & & & & & & \\
\hline $\begin{array}{l}\text { CT } 90 \\
\text { CT } 87\end{array}$ & & & & & & \\
\hline & & & & & & \\
\hline CC 43 & & & .51 & & & \\
\hline $\begin{array}{l}\text { C553 } \\
\text { CC } 56\end{array}$ & & & .48 & & & \\
\hline $\begin{array}{l}\mathrm{CC} 41 \\
\mathrm{CC} 39\end{array}$ & & & .47 & & & \\
\hline C C 7 0 & & & .46 & & & \\
\hline CC 79 & & & .44 & & & \\
\hline CC 18 & & & .43 & & & \\
\hline CC 47 & & & .40 & & & \\
\hline $\begin{array}{l}\text { CC21 } \\
\text { CC } 13\end{array}$ & & & .38 & & & \\
\hline $\begin{array}{lll}\text { CC } 51 \\
\text { C } 67\end{array}$ & & & .36 & & & \\
\hline CC 80 & & & .31 & & & \\
\hline CA 58 & & & & .55 & & \\
\hline $\begin{array}{l}\text { CA 97 } \\
\text { CA 28 }\end{array}$ & & & & .53 & & \\
\hline & & & & -.52 & & \\
\hline CA 25 & & & & .44 & & \\
\hline CA 33 & & & & -.41 & & \\
\hline $\begin{array}{l}\text { CA } 42 \\
\text { CA } 52\end{array}$ & & & & .39 & & \\
\hline CA 61 & & & & .39 & & \\
\hline CA 45 & & & & .34 & & \\
\hline 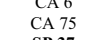 & & & & .33 & & \\
\hline $\begin{array}{l}\text { SP } 37 \\
\text { SP } 73\end{array}$ & & & & & .60 & \\
\hline $\begin{array}{l}\text { PS 82 } \\
\text { SP 59 }\end{array}$ & & & & & $\begin{array}{l}.49 \\
47\end{array}$ & \\
\hline $\begin{array}{l}\text { SP 31 } \\
\text { SP 69 }\end{array}$ & & & & & .43 & \\
\hline $\begin{array}{l}\text { SP 46 } \\
\text { SP 14 }\end{array}$ & & \multirow{3}{*}{\multicolumn{3}{|c|}{$\begin{array}{l}\text { Análisis Factorial: Método de extracción de } \\
\text { componentes principales. } \\
\text { Método de rotación, normalización Varimax con Kaiser. }\end{array}$}} & $\begin{array}{l}.32 \\
.31\end{array}$ & \\
\hline JP 19 & & & & & & \\
\hline JP 34 & & & & & & 52 \\
\hline $\mathrm{JP} 30$ & & & & & & $\begin{array}{l}31 \\
25 \\
\end{array}$ \\
\hline
\end{tabular}


El cuestionario quedó finalmente constituido por 89 reactivos de un total original de 106, de la siguiente manera:

\begin{tabular}{|l|c|}
\hline \multicolumn{1}{|c|}{ FACTORES } & ÍTEMES \\
\hline Satisfacción general en el trabajo & 24 \\
Compromiso con el trabajo & 19 \\
Compromiso de conveniencia & 17 \\
Compromiso afectivo & 16 \\
Sentimiento de permanencia & 08 \\
Jerarquía del puesto & 05 \\
\hline Total & $\mathbf{8 9}$ \\
\hline
\end{tabular}

\section{b) Fiabilidad de la escala}

De acuerdo al coeficiente alpha de Crombach los diferentes factores cumplen con el criterio de estabilidad y confiabilidad, como se puede ver en el cuadro que sigue, haciendo que el cuestionario total arroje un coeficiente $\propto=0.88$.

\section{FIABILIDAD DEL CUESTIONARIO}

\begin{tabular}{|l|c|}
\hline SUBESCALAS & Alpha de Cronbach \\
\hline Satisfacción general en el trabajo & 0.94 \\
Compromiso con el trabajo & 0.83 \\
Compromiso de conveniencia & 0.77 \\
Compromiso afectivo & 0.58 \\
Sentimiento de permanencia & 0.62 \\
Jerarquía del puesto & 0.54 \\
\hline ESCALA TOTAL & 0.88 \\
\hline
\end{tabular}

Finalmente, es preciso señalar que al cuestionario principal se le incluyó datos adicionales relacionados a la información demográfica tales como: condición laboral, sexo, edad, estudios, estado civil, antigüedad en la organización, antigüedad en el puesto, jerarquía en la organización (supervisión de otras personas y recursos), entre otros.

\section{Procedimiento}

El cuestionario fue administrado en forma colectiva y eventualmente aplicado en entrevista individual en sus lugares de trabajo a todos los trabajadores previamente selecionados al azar.

\section{Procesamiento de datos}

Se utilizó el paquete estadístico SPSS (Statistical Package for the Social Sciences) para el tratamiento estadístico. De manera especial se utilizó el estadístico de correlación de Pearson para establecer la asociación entre los diferentes factores, fueron también de utilidad la prueba «t» de Student para muestras independientes aplicada a las variables demográficas de dos grupos y el ANOVA para las variables mayores a dos grupos. 


\section{RESULTADOS}

Una evaluación general (Cuadro $\mathrm{N}^{\circ}$ 1) del compromiso organizacional de los trabajadores no docentes de una universidad pública de Lima, a través de 6 factores (satisfacción general con el trabajo, compromiso con el trabajo, compromiso de conveniencia, compromiso afectivo, sentimiento de permanencia y jerarquía del puesto), a juzgar por su media ( $\mathrm{x}=$ 4.6), muestra una tendencia favorable hacia el compromiso organizacional, confirmando en parte la hipótesis planteada. Lo que significa que existe un alto porcentaje de trabajadores administrativos que no se comprometen a plenitud con la organización dentro de los factores explorados.

Cuadro 1. Puntuacion total de compromiso organizacional

\begin{tabular}{|c|c|c|c|c|c|c|}
\hline N $^{\circ}$ CASOS & MEDIA & MEDIANA & MODA & D.S. & MÍNIMO & MÁXIMO \\
\hline 205 & 4.5566 & 4.5506 & 4.29 & .48918 & 3.40 & 5.83 \\
\hline
\end{tabular}

En el análisis de correlación de los factores de compromiso organizacional (Cuadro 2) de los trabajadores se encontró que existe correlación muy significativa y positiva entre satisfacción general con el trabajo y compromiso con el trabajo $(\mathrm{r}=.279 * *)$, compromiso afectivo $\left(\mathrm{r}=.464^{*}\right)$ y jerarquía del puesto $\left(\mathrm{r}=.407^{* *}\right)$; es decir, a mayor satisfacción general con el trabajo mayor compromiso con el trabajo, mayor compromiso afectivo y mayor compromiso con la jerarquía del puesto. También es significativa la correlación entre la satisfacción general con el trabajo y el sentimiento de permanencia en su centro de trabajo $\left(\mathrm{r}=.161^{*}\right)$, luego, a mayor satisfacción general con el trabajo mayor es el sentimiento de permanencia. Mientras tanto, la relación entre la satisfacción general con el trabajo y el compromiso de conveniencia es muy significativa pero negativa $(\mathrm{r}=-.279 * *)$; es decir, a mayor satisfacción general con el trabajo menor compromiso de conveniencia de los trabajadores.

Por otro lado, el mismo Cuadro nos muestra que entre el factor compromiso con el trabajo y compromiso de conveniencia existe una correlación muy significativa pero negativa ( $\mathrm{r}=$ $-.267 * *)$, esto es, a mayor compromiso con el trabajo menor compromiso de conveniencia. Igualmente, encontramos una asociación muy significativa y positiva entre el compromiso con el trabajo y el compromiso afectivo $\left(\mathrm{r}=.373^{* *}\right)$, sentimiento de permanencia $(\mathrm{r}=$ $\left..293^{* *}\right)$ y jerarquía del puesto $\left(\mathrm{r}=.249^{* *}\right)$, lo que significa que a mayor compromiso con el trabajo existe mayor compromiso afectivo, mayor sentimiento de permanencia y mayor compromiso con la jerarquía del puesto.

También encontramos relación muy significativa y positiva entre el compromiso afectivo y el sentimiento de permanencia, y la jerarquía del puesto; lo que quiere decir, que a mayor compromiso afectivo se da mayor sentimiento de permanencia $\left(\mathrm{r}=.355^{* *}\right)$ y mayor compromiso con la jerarquía del puesto $(\mathrm{r}=.277 * *)$.

A manera de conclusión, se puede decir que la asociación de los factores de compromiso organizacional es consistente; de allí que se muestra la tendencia favorable de los trabajadores no docentes hacia el compromiso organizacional en términos generales, sin embargo, es preciso destacar la relación entre la satisfacción general con el trabajo y el compromiso de 
conveniencia que es negativa debido probablemente a que las condiciones económicas precarias, las condiciones de inestabilidad laboral y el alto índice de desempleo estén influyendo para un comportamiento condicionado de los trabajadores. Situación similar ocurre con el factor compromiso con el trabajo y compromiso de conveniencia, y las razones pueden ser las mismas. Sin embargo, cuando la relación es entre compromiso de conveniencia y la jerarquía del puesto (aún no siendo significativa), la relación es negativa; lo que puede estar indicando que los trabajadores no muestran su compromiso con la jerarquía, probablemente porque su compromiso está condicionado a mayor satisfacción y no les preocupa ni lo afectivo, ni el compromiso moral.

Cuadro 2. Correlación de factores de compromiso organizacional

\begin{tabular}{|c|c|c|c|c|c|c|c|}
\hline \multicolumn{2}{|c|}{ FACTORES } & $\begin{array}{c}\text { Satisfacción } \\
\text { general con el }\end{array}$ & $\begin{array}{c}\text { Compromiso } \\
\text { con el }\end{array}$ & $\begin{array}{c}\text { Compromiso de } \\
\text { conveniencia }\end{array}$ & $\begin{array}{c}\text { Compromiso } \\
\text { afectivo }\end{array}$ & $\begin{array}{c}\text { Sentimiento } \\
\text { de permanencia }\end{array}$ & $\begin{array}{l}\text { Jerarquía } \\
\text { del Puesto }\end{array}$ \\
\hline $\begin{array}{l}\text { Satisfacción } \\
\text { con el trabajo }\end{array}$ & $\begin{array}{l}\text { Corr. Pearson } \\
\text { Sig (bilateral) }\end{array}$ & 1 & $\begin{array}{r}.279^{* *} \\
.000\end{array}$ & $\begin{array}{r}-.279^{* *} \\
.000\end{array}$ & $\begin{array}{r}.464^{* *} \\
.000\end{array}$ & $\begin{array}{r}.161^{*} \\
.021\end{array}$ & $\begin{array}{r}.407^{* * *} \\
.000\end{array}$ \\
\hline $\begin{array}{l}\text { Compromiso } \\
\text { con el trabajo }\end{array}$ & $\begin{array}{l}\text { Corr. Pearson } \\
\text { Sig (bilateral) }\end{array}$ & & 1 & $\begin{array}{r}-.267^{* *} \\
.000\end{array}$ & $\begin{array}{r}.373^{* *} \\
.000\end{array}$ & $\begin{array}{r}.293^{* *} \\
.000\end{array}$ & $\begin{array}{r}.249^{* *} \\
.000\end{array}$ \\
\hline $\begin{array}{l}\text { Compromiso de } \\
\text { conveniencia }\end{array}$ & $\begin{array}{l}\text { Corr. Pearson } \\
\text { Sig (bilateral) }\end{array}$ & & & 1 & $\begin{array}{l}.004 \\
.959\end{array}$ & $\begin{array}{l}.059 \\
.399\end{array}$ & $\begin{array}{r}-.089 \\
.203\end{array}$ \\
\hline $\begin{array}{l}\text { Compromiso } \\
\text { afectivo }\end{array}$ & $\begin{array}{l}\text { Corr. Pearson } \\
\text { Sig (bilateral) }\end{array}$ & & & & 1 & $\begin{array}{r}.355^{* *} \\
.000\end{array}$ & $\begin{array}{r}.277^{* *} \\
.000\end{array}$ \\
\hline $\begin{array}{l}\text { Sentimiento de } \\
\text { permanencia }\end{array}$ & $\begin{array}{l}\text { Corr. Pearson } \\
\text { Sig (bilateral) }\end{array}$ & & & & & 1 & $\begin{array}{l}.00 \\
.122 \\
.082\end{array}$ \\
\hline $\begin{array}{l}\text { Jerarquía } \\
\text { del puesto }\end{array}$ & $\begin{array}{l}\text { Corr. Pearson } \\
\text { Sig (bilateral) }\end{array}$ & & & & & & 1 \\
\hline
\end{tabular}

\footnotetext{
(*) Muy significativo .01

$\left(^{*}\right)$ Significativo .05

$\mathrm{N}=205$
}

\section{Compromiso organizacional y algunas variables demográficas}

\section{Compromiso organizacional y sexo}

En el Cuadro 3 se encuentra el análisis comparativo por sexo, respecto a compromiso organizacional. A nivel descriptivo se puede decir que participaron 205 trabajadores no docentes, de los cuales 125 fueron mujeres y obtuvieron una puntuación media de 4.54, y 80 varones que obtuvieron una puntuación media de 4.59.

A fin de conocer si existen diferencias significativas respecto a compromiso organizacional por sexo, los datos fueron sometidos a la prueba «t» de Student para muestras independientes. La prueba de Varianza, con un valor $\mathrm{F}=0.752$ y $\mathrm{P}=0.387$, nos permite afirmar que las variables son homogéneas. Con esta base se procedió a analizar la prueba de medias, obteniendo un valor de $t=-0.772$, grados de libertad g1 $=203$ y con una probabilidad de $\mathrm{P}=0.441$, permitiéndonos afirmar que no existen diferencias significativas respecto al compromiso organizacional cuando se compara hombres con mujeres.

En conclusión, entre trabajadores hombres y mujeres de una universidad pública de Lima, el compromiso organizacional es igual; en otros términos, la variable sexo no se muestra como una variable diferenciadora. 


\section{Compromiso organizacional y condiciones laborales}

En el Cuadro 3 también se encuentra el análisis comparativo por condiciones laborales, respecto a compromiso organizacional. A fin de conocer si existen diferencias significativas respecto a compromiso organizacional por condiciones laborales, los datos fueron sometidos a la prueba «t» de Student para muestras independientes. La prueba de Varianza, arrojó un valor $\mathrm{F}=2.047$ y $\mathrm{P}=0.154$ permitiéndonos afirmar que las variables son homogéneas. Con esta base se procedió a analizar la prueba de medias, obteniendo un valor de $\mathrm{t}=.098$, grados de libertad gl $=203$ y con una probabilidad de $\mathrm{P}=0.922$, llevándonos a afirmar que no existen diferencias significativas respecto al compromiso organizacional cuando se compara trabajadores contratados y nombrados.

En conclusión, la condición laboral de los trabajadores no es una variable que permita establecer diferencias respecto al compromiso organizacional; en otros términos, el compromiso organizacional es igual para trabajadores contratados o nombrados. Podría inferirse que existe una fuerte influencia de los nombrados sobre los contratados para un aprendizaje necesario y suficiente que no vaya más allá de un comportamiento similar.

Cuadro 3. Comparacion de compromiso organizacional por sexo y condiciones laborales.

\section{Compromiso organizacional y otras variables demograficas}

En los Cuadros 4 y 5, se ve el análisis comparativo de las diferentes variables demográficas (grupos de edad, estado civil, nivel máximo de estudios, número de trabajadores, antigüedad en la universidad, antigüedad en el puesto actual, puesto actual y Facultades) con respecto al compromiso organizacional. Los resultados de los participantes, fueron sometidos a la prueba ANOVA para muestras mayores a dos grupos. La prueba de Varianza arrojó que todas las variables demográficas son homogéneas $(\mathrm{P}=.774, \mathrm{P}=.667, \mathrm{P}=.132, \mathrm{P}=.853, \mathrm{P}=.349, \mathrm{P}=.349, \mathrm{P}=.425$ y $\mathrm{P}=.661$ respectivamente), así como, no se encontró diferencias significativas respecto a sus medias $(\mathrm{P}=.524, \mathrm{P}=.597, \mathrm{P}=583, \mathrm{P}=.592, \mathrm{P}=.978, \mathrm{P}=.936, \mathrm{P}=.903$ y $\mathrm{P}=.559$ respectivamente) sobre compromiso organizacional.

En conclusión, las variables demográficas señaladas en los cuadros 4 y 5 de los trabajadores no docentes, no son variables que permitan establecer diferencias respecto a compromiso organizacional; en otros términos, el compromiso organizacional es igual para todos los trabajadores a pesar de las características singulares que pueda tener cada grupo. 
Cuadro $\mathbf{N}^{\circ}$ 4. Comparación de variables demográficas sobre compromiso organizacional.

\begin{tabular}{|c|c|c|c|c|c|c|c|c|c|}
\hline \multicolumn{4}{|l|}{ VARIABLES } & \multicolumn{4}{|c|}{ PRUEBA DE VARIANZA } & \multicolumn{2}{|c|}{ ANOVA } \\
\hline & & M & D. S. & Levene & gl 1 & gl 2 & $P$ & $F$ & $P$ \\
\hline $\begin{array}{l}\text { Grupos de } \\
\text { edad }\end{array}$ & $\begin{array}{l}21-30 \\
31-40 \\
41-50 \\
51-60\end{array}$ & $\begin{array}{l}4.55 \\
4.51 \\
4.55 \\
4.67\end{array}$ & $\begin{array}{l}.46 \\
.47 \\
.54 \\
.48\end{array}$ & .372 & 3 & 201 & .774 & .749 & .524 \\
\hline Estado civil & $\begin{array}{l}\text { Soltero } \\
\text { Casado } \\
\text { Viudo } \\
\text { Divorciado } \\
\text { Unión libre } \\
\end{array}$ & $\begin{array}{l}4.57 \\
4.58 \\
4.28 \\
4.51 \\
4.48 \\
\end{array}$ & $\begin{array}{l}.46 \\
.52 \\
.50 \\
.34 \\
.45 \\
\end{array}$ & .595 & 4 & 200 & .667 & .694 & .597 \\
\hline $\begin{array}{l}\text { Nivel máximo } \\
\text { de estudios }\end{array}$ & $\begin{array}{l}\text { Secundaria } \\
\text { Técnico } \\
\text { Superior In } \\
\text { Superior co } \\
\text { Profesional } \\
\text { Post Grado }\end{array}$ & $\begin{array}{l}4.58 \\
4.57 \\
4.57 \\
4.62 \\
4.39 \\
4.44\end{array}$ & $\begin{array}{l}.38 \\
.55 \\
.48 \\
.45 \\
.48 \\
.66\end{array}$ & 1.720 & 5 & 199 & .132 & .756 & 583 \\
\hline $\begin{array}{l}\text { Número de } \\
\text { trabajadores }\end{array}$ & $\begin{array}{l}\text { Menos } 25 \\
26-50 \\
51-100 \\
101-200 \\
201-500 \\
\end{array}$ & $\begin{array}{l}4.70 \\
4.58 \\
4.56 \\
4.49 \\
4.38 \\
\end{array}$ & $\begin{array}{l}.46 \\
.49 \\
.53 \\
.44 \\
.42 \\
\end{array}$ & .337 & 4 & 200 & .853 & .701 & .592 \\
\hline $\begin{array}{l}\text { Antigüedad en } \\
\text { la universidad }\end{array}$ & $\begin{array}{l}\text { Menos de } 1 \\
1 \text { a } 5 \text { años } \\
6 \text { a } 10 \\
11 \text { a } 12 \\
21 \text { a } 30 \\
\text { Mas de } 30\end{array}$ & $\begin{array}{l}4.52 \\
4.54 \\
4.58 \\
4.58 \\
4-52 \\
4.66\end{array}$ & $\begin{array}{l}.43 \\
.50 \\
.46 \\
.54 \\
.47 \\
.21\end{array}$ & 1.124 & 4 & 199 & .349 & .155 & .978 \\
\hline $\begin{array}{l}\text { Antigüedad } \\
\text { puesto actual }\end{array}$ & $\begin{array}{l}\text { Menos de } 1 \\
1 \text { a } 5 \text { años } \\
6 \text { a } 10 \\
11 \text { a } 12 \\
21 \text { a } 30 \\
\text { Mas de } 30\end{array}$ & $\begin{array}{l}4.57 \\
4.53 \\
4.56 \\
4-64 \\
4.55 \\
4.81\end{array}$ & $\begin{array}{l}.52 \\
.47 \\
.47 \\
.59 \\
.26\end{array}$ & 1.044 & 4 & 199 & . 386 & .256 & .936 \\
\hline Puesto actual & $\begin{array}{l}\text { Director, Je } \\
\text { Funciona II } \\
\text { Funcionari I } \\
\text { Empl o Téc } \\
\text { Trab.manual } \\
\text { Otros }\end{array}$ & $\begin{array}{l}4.64 \\
4.56 \\
4.58 \\
4.58 \\
4.51 \\
4.39\end{array}$ & $\begin{array}{l}.81 \\
.49 \\
.63 \\
.47 \\
.51 \\
.49\end{array}$ & .989 & 5 & 198 & .425 & .361 & .903 \\
\hline
\end{tabular}


Cuadro $\mathbf{N}^{0}$ 5. Comparación de la variable facultades sobre compromiso organizacional.

\begin{tabular}{|c|c|c|c|c|c|c|c|c|c|}
\hline \multicolumn{2}{|l|}{ VARIABLES } & & \multirow{2}{*}{$\begin{array}{c}\text { D. S. } \\
.45\end{array}$} & \multicolumn{4}{|c|}{ PRUEBA DE VARIANZA } & \multicolumn{2}{|c|}{ ANOVA } \\
\hline & & & & Levene & gl 1 & gl 2 & $\mathbf{P}$ & $\mathbf{F}$ & $\mathbf{P}$ \\
\hline FACULTADES & $\begin{array}{l}\text { Química } \\
\text { Matemáticas } \\
\text { Física } \\
\text { Biología } \\
\text { Veterinaria } \\
\text { CC. Sociales } \\
\text { Administración } \\
\text { Contabilidad } \\
\text { Economía } \\
\text { Letras } \\
\text { Educación } \\
\text { Psicología } \\
\text { Electrónica } \\
\text { Ing. Industrial } \\
\text { Geología } \\
\text { Medicina } \\
\text { Farmacia } \\
\text { Ing. Sistema } \\
\text { Odontología } \\
\text { Derecho }\end{array}$ & $\begin{array}{l}4.63 \\
4.62 \\
4.57 \\
4.56 \\
4.33 \\
4.75 \\
4.82 \\
4-50 \\
4.50 \\
4.74 \\
4.54 \\
4.35 \\
4.40 \\
4.56 \\
4.81 \\
4.37 \\
4.66 \\
4.46 \\
4.60 \\
4.45 \\
\end{array}$ & $\begin{array}{l}.45 \\
.40 \\
.46 \\
.63 \\
.45 \\
.43 \\
.66 \\
.50 \\
.44 \\
.44 \\
.49 \\
.49 \\
.32 \\
.58 \\
.53 \\
.43 \\
.39 \\
.40 \\
.46 \\
.70 \\
\end{array}$ & .837 & 19 & 185 & .661 & .920 & .559 \\
\hline
\end{tabular}

\section{Factores de compromiso organizacional y variables sociodemográficas}

En los Cuadros que van del 6 al 15 (ver anexo en el informe ampliado) se encuentran los análisis comparativos entre los factores de compromiso organizacional y las variables sociodemográficas, tales como sexo, condiciones laborales, edad, estado civil, nivel máximo de estudios, número de trabajadores, antigüedad en la universidad, antigüedad en el puesto actual, puesto actual y Facultades. Para conocer sus diferencias significativas fueron sometidas a las pruebas t de Student y Anova según el caso.

\section{Factores de compromiso organizacional por sexo}

En el Cuadro 6 se encuentra el análisis comparativo entre satisfacción general en el trabajo y sexo, la media obtenida por el grupo de hombres fue de 4.28, y por el grupo de mujeres, una media de 3.97. A fin de conocer si existen diferencias significativas, los datos fueron sometidos a la prueba «t» de Student para muestras independientes. La prueba de Varianza arrojó un valor $\mathrm{F}=0.004$ y una probabilidad de $\mathrm{P}=0.952$ permitiéndonos afirmar que son homogéneas. Con esta base se procedió a analizar la prueba de medias, obteniendo un valor de $\mathrm{t}=-1.852$, grados de libertad $\mathrm{gl}=203 \mathrm{y}$ con una probabilidad de $\mathrm{P}=0.065$, llevándonos a afirmar que no existen diferencias significativas entre hombres y mujeres respecto al compromiso organizacional. Sin embargo, por la cercanía de dicha probabilidad al convencional (0.05) se puede asumir que existe una tendencia a diferenciarse entre ambos grupos respecto a la satisfacción general en el trabajo. Y en este caso particular, son los hombres los que muestran una mayor satisfacción comparado con las mujeres. 
En conclusión, el sexo de los trabajadores, no es una variable que permita establecer diferencias respecto a la satisfacción general en el trabajo como componente del compromiso organizacional; aunque hay una tendencia de satisfacción más alta en los hombres que en las mujeres. Este hecho puede estar en relación con el tipo de responsabilidad asumida en el trabajo.

En el análisis comparativo por sexo, respecto a los demás factores del Compromiso Organizacional, tales como compromiso con el trabajo $(\mathrm{P}=0.093)$, compromiso afectivo $(\mathrm{P}=0.959)$ y sentimiento de permanencia $(\mathrm{P}=0.524)$, se encontró resultados similares que en el factor anterior, donde no hay diferencias significativas, salvo una ligera tendencia más alta de compromiso con el trabajo por parte de las mujeres que los hombres. Esto puede estar indicando que, a pesar de no estar plenamente satisfecho en el trabajo, hay una tendencia de las mujeres a estar más comprometida con el trabajo que los hombres.

Por otro lado, en el análisis comparativo por sexo, respecto a los factores del Compromiso Organizacional, tales como, compromiso de conveniencia $(\mathrm{F}=4.172$; $\mathrm{P}=0.042$ y $\mathrm{t}=0.037 ; \mathrm{P}=0.970)$ y jerarquía del puesto $(\mathrm{F}=5.744 ; \mathrm{P}=0.017 \mathrm{y} \mathrm{t}=-1.143$ y $\mathrm{P}=0.254)$, se encontró que no existen diferencias significativas entre las puntuaciones procedente de los hombres y mujeres. Es decir, hombres y mujeres muestran una tendencia similar frente al compromiso de conveniencia y la jerarquía del puesto.

\section{Factores de compromiso organizacional por condiciones laborales}

En el Cuadro 7 se observa el análisis comparativo por condiciones laborales respecto a los factores del Compromiso Organizacional, tales como satisfacción general en el trabajo $(\mathrm{t}=0.127$ y $\mathrm{P}=0.899)$, compromiso con el trabajo $(\mathrm{t}=0.638$ y $\mathrm{P}=0.524)$, compromiso de conveniencia $(\mathrm{t}=-0.487$ y $\mathrm{P}=0.627)$, compromiso afectivo $(\mathrm{t}=-1.088 \mathrm{y}$ $\mathrm{P}=0.278)$, sentimiento de permanencia $(\mathrm{t}=1.318$ y $\mathrm{P}=0.189)$ y jerarquía del puesto $(\mathrm{t}=-0.093$ y $\mathrm{P}=0.926)$, que nos muestra que no existen diferencias significativas respecto a los factores del compromiso organizacional cuando se comparan a grupos de trabajadores de acuerdo a las condiciones laborales (contratados y nombrados).

En conclusión, se puede decir que las condiciones laborales (contratados y nombrados) no son variables que permitan distinguir diferencias sobre los factores de compromiso organizacional. Son homogéneos frente a los diferentes componentes del compromiso organizacional.

\section{Factores de compromiso organizacional por grupos de edad}

En el Cuadro 8 se ve el análisis comparativo de los grupos de edad, respecto a los factores del compromiso organizacional (satisfacción general en el trabajo, compromiso con el trabajo, compromiso de conveniencia, compromiso afectivo, sentimiento de permanencia y jerarquía del puesto). Los resultados obtenidos de los trabajadores, fueron sometidos a la prueba ANOVA para muestras mayores a dos grupos. En ella, la prueba de varianza arrojó que todos los grupos de edad son homogéneos $(\mathrm{P}=.877$, $\mathrm{P}=.901, \mathrm{P}=.710, \mathrm{P}=.871, \mathrm{P}=.881$ y $\mathrm{P}=.918$, respectivamente) y no existen diferencias significativas respecto a los factores de compromiso organizacional $(\mathrm{P}=.069, \mathrm{P}=.799$, $\mathrm{P}=.961, \mathrm{P}=.669, \mathrm{P}=.212$ y $\mathrm{P}=.881$, respectivamente). 
En conclusión, los grupos de edad señalados en el cuadro indicado, de los trabajadores no docentes, no son variables que permitan establecer diferencias respecto a los factores de compromiso organizacional; en otros términos, el compromiso organizacional es igual para todos los trabajadores a pesar de las características singulares que pueda tener cada grupo. Sin embargo, existe una ligera tendencia a elevarse la satisfacción general en el trabajo de los trabajadores de 51 a 60 años. Tal vez por que a ésa edad han logrado cierta estabilidad en el puesto, las expectativas tienden a disminuir o han ganado cierta imagen que les hace sentir seguros.

\section{Factores de compromiso organizacional por estado civil}

En el Cuadro $\mathrm{N}^{\circ}$ 9, se ve el análisis comparativo por estado civil respecto a los factores del compromiso organizacional (satisfacción general en el trabajo, compromiso con el trabajo, compromiso de conveniencia, compromiso afectivo, sentimiento de permanencia y jerarquía del puesto). Los resultados obtenidos en los participantes fueron sometidos a la prueba ANOVA. Así, en la prueba de varianza se mostraron homogéneos $(\mathrm{P}=.101, \mathrm{P}=.304, \mathrm{P}=.847, \mathrm{P}=.825, \mathrm{P}=.803$ y $\mathrm{P}=.716$ respectivamente); y no se encontró diferencias significativas respecto a sus medias ( $\mathrm{P}$ $=.578, \mathrm{P}=.849, \mathrm{P}=.152, \mathrm{P}=.855, \mathrm{P}=.118$ y $\mathrm{P}=.471$ respectivamente) en ninguno de los factores de compromiso organizacional.

En conclusión, el estado civil de los trabajadores, no se muestra como variable diferenciadora respecto a los diversos factores de compromiso organizacional.

\section{Factores de compromiso organizacional por nivel maximo de estudios}

En el Cuadro $\mathrm{N}^{\circ} 10$, se ve el análisis comparativo por niveles máximos de estudio respecto a los factores del compromiso organizacional (satisfacción general en el trabajo, compromiso de conveniencia, compromiso afectivo y jerarquía del puesto). Sometidos los datos, a la prueba ANOVA para muestras mayores a dos grupos, se encontró que son grupos homogéneos $(\mathrm{P}=.015, \mathrm{P}=.225, \mathrm{P}=.350$ y $\mathrm{P}=.136$, respectivamente), y no existen diferencias significativas $(\mathrm{P}=.179, \mathrm{P}=.198, \mathrm{P}=.890 \mathrm{y}$ $\mathrm{P}=.470$, respectivamente) respecto a los factores de compromiso organizacional.

En conclusión, el nivel de estudios de los trabajadores no docentes, no son variables que permitan establecer diferencias respecto a algunos factores de compromiso organizacional; en otros términos, los trabajadores se comportan de manera similar.

En cambio, el análisis comparativo del mismo cuadro nos muestra a través de la prueba ANOVA, que los grupos por nivel máximo de estudios son heterogéneos con respecto a compromiso con el trabajo y sentimiento de permanencia $(\mathrm{P}=.292$ y $\mathrm{P}=.191$, respectivamente), permitiéndonos afirmar que existen diferencias significativas $(\mathrm{P}=.042$ y $\mathrm{P}=.013$, respectivamente). Respecto al primer caso, los trabajadores con educación superior completa obtienen una media más alta $(\mathrm{X}=6.08)$ que los demás grupos; y respecto al segundo caso, los trabajadores con estudios de post grado obtienen una media más alta $(X=5.54)$ que los demás.

En conclusión, los niveles máximos de estudio de los trabajadores son variables que nos permiten establecer diferencias entre los grupos respecto a los factores de 
compromiso con el trabajo y sentimiento de permanencia; es decir, el compromiso con el trabajo es más alto en trabajadores con educación superior completa que los demás grupos y, también el sentimiento de permanencia es más alto en relación con los demás, en trabajadores que han hecho estudios de post grado. Probablemente por que los estudios superiores les permite asumir funciones de mayor jerarquía y comprender el grado de responsabilidad del puesto.

\section{Factores de compromiso organizacional por numero de trabajadores en su unidad de trabajo}

En el Cuadro 11, se ve el análisis comparativo por número de trabajadores en la unidad donde labora respecto a los factores del compromiso organizacional (satisfacción general en el trabajo, compromiso con el trabajo, compromiso de conveniencia, compromiso afectivo, sentimiento de permanencia y jerarquía del puesto). Sometidos los datos obtenidos de los participantes a la prueba ANOVA para muestras mayores a dos grupos, se encontró que son grupos homogéneos $(\mathrm{P}=.304, \mathrm{P}=.411, \mathrm{P}=.428, \mathrm{P}=.974$, $\mathrm{P}=.856$ y $\mathrm{P}=.674$ respectivamente), y no existen diferencias significativas respecto a los factores de compromiso organizacional mencionados $(\mathrm{P}=.760, \mathrm{P}=.483, \mathrm{P}=.361$, $\mathrm{P}=.249, \mathrm{P}=.091$ y $\mathrm{P}=.634)$. Sin embargo, aquellos trabajadores que laboran en una unidad con menos de 25 trabajadores son los que obtienen una media más alta $(\mathrm{x}=5.00)$ en sentimiento de permanencia.

En conclusión, la cantidad de trabajadores en el lugar de trabajo de los encuestados, como variables de análisis no ayuda para establecer diferencias respecto a los factores de compromiso organizacional; en otros términos, los trabajadores se comportan de manera similar. Salvo aquellos trabajadores que saben que hay pocos trabajadores en su lugar de trabajo que tienen una tendencia más alta respecto al sentimiento de permanencia, puede deberse al sentido del deber o la reciprocidad para con la organización.

\section{Factores de compromiso organizacional por antigüedad en la universidad}

En el Cuadro 12, se ve el análisis comparativo por antigüedad en la organización respecto a los factores del compromiso organizacional (satisfacción general en el trabajo, compromiso con el trabajo, compromiso afectivo, sentimiento de permanencia y jerarquía del puesto). Sometidos los resultados de los participantes a la prueba ANOVA para muestras mayores a dos grupos, se encontró que son grupos homogéneos $(\mathrm{P}=.712, \mathrm{P}=.111, \mathrm{P}=.334, \mathrm{P}=.032$ y $\mathrm{P}=.708$, respectivamente $)$ y no existen diferencias significativas $(\mathrm{P}=.511, \mathrm{P}=.560, \mathrm{P}=.186, \mathrm{P}=.269$ y $\mathrm{P}=.888$, respectivamente) respecto a los factores de compromiso organizacional.

En conclusión, las variables por antigüedad de los trabajadores en la organización, no permiten establecer diferencias respecto a algunos factores de compromiso organizacional; en otros términos, los trabajadores se comportan de manera similar.

Sin embargo, en el factor compromiso de conveniencia los años de servicio tienen especial importancia. El análisis comparativo del mismo cuadro nos muestra a través de la prueba ANOVA, que los grupos son heterogéneos $(\mathrm{P}=.707)$, permitiéndonos 
afirmar que existen diferencias significativas $(\mathrm{P}=0.043)$ entre los grupos por años de servicio; siendo más alto la media $(X=3.79)$ del grupo de trabajadores que tiene entre 11 y 20 años de servicio.

En conclusión, los años de servicio de los trabajadores en la organización nos permite establecer diferencias de opinión respecto al compromiso de conveniencia; es decir, el compromiso de conveniencia es más alto en trabajadores con años de servicio entre 11 y 20 años que los demás grupos. Puede entenderse como una etapa donde los cálculos de inversión-beneficio llegan a su máximo nivel de expresión, los reclamos y los deseos de crecimiento acelerado pueden ser comunes.

\section{Factores de compromiso organizacional por antigüedad en el puesto actual}

En el Cuadro 13, se ve el análisis comparativo por antigüedad en el puesto actual respecto a los factores del compromiso organizacional (satisfacción general en el trabajo, compromiso con el trabajo, compromiso afectivo, sentimiento de permanencia y jerarquía del puesto). Sometidos los resultados de los participantes, a la prueba ANOVA para muestras mayores a dos grupos, se encontró que son grupos homogéneos $(\mathrm{P}=.698, \mathrm{P}=.433, \mathrm{P}=.793, \mathrm{P}=.061$ y $\mathrm{P}=.903$, respectivamente $), \mathrm{y}$ no existen diferencias significativas $(\mathrm{P}=.500, \mathrm{P}=.372, \mathrm{P}=. .593, \mathrm{P}=.528$ y $\mathrm{P}=570$, respectivamente) respecto a los factores de compromiso organizacional señalados.

En conclusión, las variables por antigüedad de los trabajadores en el puesto que ocupan en el momento actual, no permiten establecer diferencias respecto a algunos factores de compromiso organizacional; en otros términos, los trabajadores se comportan iguales.

En cambio, en el factor compromiso de conveniencia los años de permanencia en el puesto de trabajo actual tienen especial importancia. El análisis comparativo del mismo cuadro nos muestra a través de la prueba ANOVA, que los grupos son heterogéneos $(\mathrm{P}=.416)$, permitiéndonos afirmar en este caso que existen diferencias significativas $(\mathrm{P}=0.039)$ entre los grupos por años de servicio en el puesto actual; siendo más alto la media $(X=4.22)$ del grupo de trabajadores que tiene entre 21 y 30 años de servicio.

En conclusión, los años de servicio laborados en el puesto actual nos permiten establecer diferencias respecto al compromiso de conveniencia; es decir, el compromiso de conveniencia es más alto en trabajadores con años de servicio en el puesto actual entre 21 y 30 años que los demás grupos. Este resultado estaría indicando el costo que significaría dejar la organización en esta etapa de su vida después de una inversión de tiempo y esfuerzo.

\section{Factores de compromiso organizacional por puesto actual}

En el Cuadro 14, se observa el análisis comparativo entre el puesto actual que ocupa el trabajador y su relación con los factores del compromiso organizacional (satisfacción general en el trabajo, compromiso con el trabajo, compromiso afectivo, sentimiento de permanencia y jerarquía del puesto). Sometidos los resultados de los participantes, a la prueba ANOVA para muestras mayores a dos grupos, se encontró que son grupos 
homogéneos $(\mathrm{P}=.691, \mathrm{P}=. .394, \mathrm{P}=.862, \mathrm{P}=.247$ y $\mathrm{P}=.720$, respectivamente $)$, y no existen diferencias significativas respecto a los factores de compromiso organizacional señalados $(\mathrm{P}=.702, \mathrm{P}=.318, \mathrm{P}=.126, \mathrm{P}=.485$ y $\mathrm{P}=.414)$.

En conclusión, la variable puesto actual no permite establecer diferencias respecto a los factores de compromiso organizacional señalados antes; en otros términos, los trabajadores se comportan de la misma manera cualquiera que fuera su puesto de trabajo.

En cambio, en el factor compromiso de conveniencia, el puesto de trabajo actual tienen especial relevancia. El análisis comparativo del mismo cuadro nos muestra a través de la prueba ANOVA, que los grupos son heterogéneos $(\mathrm{P}=.154)$, permitiéndonos afirmar que en este caso existen diferencias significativas $(\mathrm{P}=0.048)$ entre los grupos de los diferentes puestos de trabajo actuales; siendo más alto la media $(x=4.20)$ del grupo de trabajadores que ocupan puestos de dirección o jefaturas de unidad.

En conclusión, el puesto de trabajo de los trabajadores permite establecer diferencias respecto al compromiso de conveniencia; es decir, el compromiso de conveniencia es más alto en trabajadores que ocupan puestos de dirección o jefaturas de unidad, probablemente por que son cargos de confianza y les reporta mayores ingresos económicos, por lo mismo estarían dispuestos a asumir los riesgos de la estabilidad.

\section{Factores de compromiso organizacional por areas de trabajo o facultades}

En el Cuadro 15, se encuentra el análisis comparativo por Facultades respecto a los factores del compromiso organizacional (satisfacción general en el trabajo, compromiso con el trabajo, compromiso de conveniencia, compromiso afectivo, sentimiento de permanencia y jerarquía del puesto). Los grupos de participantes fueron sometidos a la prueba ANOVA para muestras mayores a dos grupos, encontrándose que son grupos homogéneos $(\mathrm{P}=.093, \mathrm{P}=.383, \mathrm{P}=.932, \mathrm{P}=.904, \mathrm{P}=.518, \mathrm{P}=.125$, respectivamente $)$ $\mathrm{y}$ no existen diferencias significativas respecto a los factores de compromiso organizacional señalados $(\mathrm{P}=.523, \mathrm{P}=.255, \mathrm{P}=.685, \mathrm{P}=.342, \mathrm{P}=.715, \mathrm{P}=.373)$.

En conclusión, la variable Facultad o área donde laboran los trabajadores no permite establecer diferencias respecto a ninguno de los factores de compromiso organizacional; en otros términos, los trabajadores se comportan del mismo modo sin importar la Facultad donde laboral o que pertenecen.

Sin embargo, se puede decir que hay una ligera tendencia a distinguirse el compromiso con el trabajo de los trabajadores de la Facultad de Administración $(x=6.38)$, seguido de trabajadores de Matemáticas $(\mathrm{x}=6.20)$ y Biología $(\mathrm{x}=6.18)$; posiblemente de debe a la influencia de las características particulares de la profesión de los profesores o al posesionamiento de la profesión en el mercado. 


\section{DISCUSIÓN}

En términos generales, los resultados nos muestran una tendencia favorable de los trabajadores hacia el compromiso organizacional, y abriendo la posibilidad de un comportamiento comprometido con la organización, en concordancia con la Teoría de Azjen y Fishbein (1977, 1980, en Arias, 2003) que señala que la intención es la antesala del comportamiento. No obstante, hay un porcentaje de trabajadores que merecen especial atención en tanto no muestran compromiso alguno con la organización.

Respecto a las correlaciones entre los factores del compromiso organizacional, la satisfacción general en el trabajo mostró asociación positiva con compromiso con el trabajo, con compromiso afectivo y jerarquía del puesto; igual hubo correlación entre el primero y el sentimiento de permanencia, aunque en menor grado, confirmando así los hallazgos de Arias, F.; Valega, D.; Loli, A. y Quintana, M. (2003) Mientras que, la relación entre la satisfacción general en el trabajo y el compromiso de conveniencia es muy significativa pero negativa, igual que el compromiso con el trabajo y compromiso de conveniencia. Estas dos últimas asociaciones negativas pueden estar en relación con las condiciones económicas y las condiciones laborales que se les ofrece y el alto índice de desempleo del mercado laboral, comportamiento coherente con la revisión de las investigaciones practicadas y su propuesta del compromiso calculador de Mathieu y Zajac (1990), los hallazgos de Bayona, C., y otros (2000) y la teoría de Meyer y Allen (1991) en el sentido de condicionar su desempeño al costo de su inversión (Becker, 1960) o a la presión del desempleo del mercado (Vandenberghe, 1996).

Por otro lado, tampoco en este estudio encontramos correlación significativa del compromiso de conveniencia o continuidad con el compromiso afectivo, con el sentimiento de permanencia ni con jerarquía del puesto (Bayona, C., y otros, 2000). No obstante, se debe señalar que la relación del primero con jerarquía del puesto es negativa, probablemente por que los trabajadores no muestran su compromiso con la jerarquía, debido a que su compromiso está condicionado a mayor satisfacción y no les preocupa el compromiso afectivo (o actitudinal de Mathieu y Zajac, 1990; o identificación de Claure, M. y Böhrt, M. 2003 y otras propuestas), ni el sentimiento de permanencia (o normativo en el lenguaje de Meyer y Allen, 1991).

Respecto a la asociación entre el compromiso organizacional y las variables demográficas, no se encuentra ninguna relación importante. Mientras tanto, un análisis con cada uno de los factores o componentes del compromiso organizacional y las variables demográficas nos permitió encontrar que existe diferencias significativas del compromiso de conveniencia con antigüedad en la organización siendo más alto el compromiso del grupo de trabajadores que tenían entre 11 a 20 años de servicios; también hubo relación con antigüedad en el puesto actual siendo más alto el compromiso del grupo de 21 a 30 años de servicio, y con el puesto que ocupa actualmente, donde hay mayor compromiso de los trabajadores que ocupan puestos de dirección o jefaturas; lo cual es consistente en parte con la característica calculadora del compromiso de conveniencia encontrado por Mathieu y Zajac (1990). También el nivel máximo de estudios exhibió relación con el compromiso con el trabajo, destacando con mayor compromiso aquellos que tenían estudios de educación superior completa, y, finalmente, aquellos que tenían estudios de postgrado exhibieron más altos sentimientos de permanencia o compromiso normativo, en contraposición con los hallazgos de Mathieu y Zajac (1990). 
Por lo demás, la edad, el sexo, el estado civil, las condiciones laborales, el número de trabajadores y la ubicación o área de trabajo no son variables útiles para establecer diferencias con los componentes del compromiso organizacional; es decir, el comportamiento de los trabajadores es similar y la presencia de estas variables no altera su compromiso con la organización.

\section{CONCLUSIONES}

1. La correlación de los factores de compromiso organizacional es consistente; de allí que se muestra la tendencia favorable de los trabajadores no docentes hacia el compromiso organizacional en términos generales; sin embargo, es preciso destacar la relación entre la satisfacción general con el trabajo y el compromiso de conveniencia que es negativa debido probablemente a que las condiciones económicas precarias, las condiciones de inestabilidad laboral y el alto índice de desempleo estén influyendo para un comportamiento condicionado de los trabajadores. Situación similar ocurre con el factor compromiso con el trabajo y compromiso de conveniencia, y las razones pueden ser las mismas. Sin embargo, cuando la relación es entre compromiso de conveniencia y la jerarquía del puesto (aún no siendo significativa), la relación es negativa; lo que puede estar indicando que los trabajadores no muestran su compromiso con la jerarquía, probablemente por que su compromiso está condicionada a mayor satisfacción y no les preocupa el compromiso afectivo.

2. Las variables tales como sexo, condiciones laborales, grupos de edad, estados civil, nivel máximo de estudios, número de trabajadores, antigüedad en la universidad, antigüedad en el puesto actual, puesto actual, área de trabajo (Facultades), no son variables que permitan establecer diferencias respecto al compromiso organizacional; en otros términos, el compromiso organizacional es igual para los trabajadores a pesar de las características singulares que pueda tener cada grupo.

3. El análisis de los factores del compromiso organizacional en relación con las variables demográficas, permitió llegar a las siguientes conclusiones:

a. El sexo de los trabajadores no es una variable que permita establecer diferencias respecto a la satisfacción general en el trabajo como componente del compromiso organizacional; aunque hay una tendencia de satisfacción más alta en los hombres que en las mujeres. Este hecho puede estar en relación con el tipo de responsabilidad asumida en el trabajo. Sin embargo, cuando se habla del compromiso con el trabajo hay una tendencia de las mujeres a estar más comprometidas con el trabajo que los hombres, a pesar de no estar plenamente satisfechas en el trabajo en general. En cambio, respecto al compromiso afectivo, sentimientos de permanencia, compromiso de conveniencia y la jerarquía del puesto hombres y mujeres muestran una tendencia similar.

b. Las condiciones laborales de los trabajadores no son variables que permitan distinguir diferencias sobre los factores del compromiso organizacional. Contratados y nombrados se comportan de la misma manera, son homogéneos frente a los diferentes componentes del compromiso organizacional.

c. Los trabajadores por grupos de edad, no son variables que permitan establecer diferencias respecto a los factores de compromiso organizacional, a pesar de las 
características singulares que pueda tener cada grupo. Sin embargo, existe una ligera tendencia a elevarse la satisfacción general en el trabajo de los trabajadores de 51 a 60 años, tal vez por que a ésa edad han logrado estabilidad en el puesto, las expectativas tienden a disminuir o han ganado cierta imagen que les hace sentir seguros.

d. El estado civil de los trabajadores no se muestra como variable diferenciadora respecto a los diversos factores de compromiso organizacional.

e. El nivel máximo de estudios de los trabajadores tampoco es variable que permitan establecer diferencias respecto a satisfacción general en el trabajo, compromiso de conveniencia, compromiso afectivo y jerarquía del puesto. Sin embargo, respecto al compromiso con el trabajo y sentimiento de permanencia, es posible encontrar deferencias: los trabajadores con educación superior completa tienen el compromiso con el trabajo más alto que los demás grupos, y los trabajadores que han hecho estudios de postgrado también tienen el sentimiento de permanencias más alto en relación con los demás. Probablemente porque los estudios superiores les permite asumir funciones de mayor jerarquía y comprender el grado de responsabilidad del puesto.

f. El número de trabajadores en el lugar de trabajo de los encuestados, como variables de análisis no ayuda para establecer diferencias respecto a los factores de compromiso organizacional. Salvo aquellos trabajadores que saben que hay pocos trabajadores en su lugar de trabajo que tienen una tendencia más alta respecto al sentimiento de permanencia, puede deberse al sentido del deber o la reciprocidad para con la organización.

g. La antigüedad de los trabajadores en la organización no permite establecer diferencias respecto a satisfacción general en el trabajo, compromiso con el trabajo, compromiso afectivo, sentimiento de permanencia y jerarquía del puesto. Sin embargo, respecto al compromiso de conveniencia, los trabajadores que tienen entre 11 y 20 años de servicio están más comprometidos que los demás grupos. Puede entenderse como una etapa donde los cálculos de inversión-beneficio llegan a su máximo nivel de expresión, los reclamos y los deseos de crecimiento acelerado pueden ser comunes.

h. La antigüedad de los trabajadores en el puesto que ocupan en el momento actual, no permite establecer diferencias respecto a satisfacción general en el trabajo, compromiso con el trabajo, compromiso afectivo, sentimiento de permanencia y jerarquía del puesto. Sin embargo, cuando se trata del compromiso de conveniencia, se da la diferencia siendo éste más alto en trabajadores con años de servicio entre 21 y 30 años que los demás grupos. Este resultado estaría indicando el costo que significaría dejar la organización en esta etapa de su vida después de una inversión de tiempo y esfuerzo.

i. El puesto actual, es una variable que no permite establecer diferencias respecto a satisfacción general en el trabajo, compromiso con el trabajo, compromiso afectivo, sentimiento de permanencia y jerarquía del puesto. Pero tratándose del compromiso de conveniencia, encontramos diferencias siendo más alto este compromiso en trabajadores que ocupan puestos de dirección o jefaturas de unidad, probablemente 
porque son cargos de confianza y les reporta mayores ingresos económicos, por lo mismo estarían dispuestos a asumir los riesgos de la estabilidad.

j. La variable Facultad o área donde laboran los trabajadores no ayuda a establecer diferencias respecto a ninguno de los factores de compromiso organizacional. Sin embargo, hay una ligera tendencia a distinguirse el compromiso con el trabajo de los trabajadores de la Facultad de Administración, seguido de trabajadores de Matemáticas y luego Biología, posiblemente de debe a la influencia de las características particulares de la profesión de los profesores o al posesionamiento de la carrera profesional en el mercado.

\section{REFERENCIAS BIBLIOGRÁFICAS}

1. Antón, C. y Gonzáles, L. (2005). Examen de las relaciones entre el compromiso organizacional afectivo y continuo. España: Universidad de Salamanca.

2. Arias Galicia, F. (2001). «El compromiso personal hacia la organización y la intención de permanencia: algunos factores para su incremento». México: Edit. UNAM. Rev. de Contaduría y Administración No 200, enero - marzo 2001.

3. Arias Galicia, F., Varela Sosa, D, Loli Pineda, A. y Quintana Otiniano, M. (2003). El compromiso organizacional y su relación con algunos factores demográficos. Rev. de Investigación en Psicología, vol. 6, $\mathrm{N}^{\circ}$ 2, UNMSM.

4. Arias Galicia, Fernando (1972). Administración de Recursos Humanos. México: Edit Trillas.

5. Arias Galicia y Heredia Espinoza (1999). Administración de Recursos Humanos para el alto Desempeño. México: Trillas.

6. Arciniega, Luis (s/f). Compromiso organizacional en México: ¿Qué es lo que hace que la gente se ponga la camiseta?. (sin información formal).

7. Bayona, C., Goñi, S. y Madorrán, C., (2000). Compromiso organizacional: implicaciones para la gestión estratégica de los recursos humanos. En: www.upna.es

8. Claure Rocha, M. y Böhrt Pelaez, M. (2003). Tres dimensiones del compromiso organizacional: identificación, membresía y lealtad. Bolivia: Rev. AJAYU, año 2003, Vol. 2, $\mathrm{N}^{\mathrm{o}}$ 1. Estas dimensiones son equivalentes a compromiso afectivo, continuidad y normativo de Meyer y Allen (1991), respectivamente.

9. Dessler, G, (1996). Administración de personal. México: Edit. Prentice-Hall Hispanoamericana S.A.

10. Gibson, J. L., Ivanovich, J.M. y Donnelly, J.H.(1994). Las organizaciones. Estados Unidos: Edit. Addison-Wesley Iberoamericana.

11. Lagomarsino, Raúl (2003). Compromiso organizacional. Uruguay: Ed. Universidad de Montevideo-IEEM año 6, No 2, agosto 2003.

12. Smith, P.C. (1976). «Desarrollo de un método para medir la satisfacción en el trabajo: los estudios de Cornell». En Fleishman, E.A. y Bass, A.R. (1976). Estudios de psicología industrial y del personal. México: Trillas. 
13. Meyer, J.P. y Allen, N.J. (1991). «Tres componentes, Conceptualización del compromiso organizacional». Human Resource Management Review, 1, 61-98.

14. Steers, R.M. (1977). «Antecedentes y resultados del compromiso organizacional». En: Administrative Science Quarterly, 22, 46-56.

15. Varona Madrid, Federico (1993). «Conceptualización y supervisión de la comunicación y el compromiso organizacional». San José, Costa Rica. Ed. FELAFACS, En: Rev. Diálogos No 35 , pp. 68-77.

\section{ANEXOS}

Cuadro 6. Comparación de factores de compromiso organizacional por sexo.

\begin{tabular}{|c|c|c|c|c|c|c|c|c|}
\hline \multirow[t]{2}{*}{ VARIABLES } & \multirow[b]{2}{*}{ SEXO } & \multirow[b]{2}{*}{ M } & \multirow[b]{2}{*}{ D. S. } & \multicolumn{2}{|c|}{ Prueba de Varianza } & \multicolumn{3}{|c|}{ Prueba de Medias } \\
\hline & & & & $\mathbf{F}$ & $\mathbf{P}$ & t & gl & $\mathbf{p}$ \\
\hline $\begin{array}{l}\text { Satisfacción } \\
\text { general en el } \\
\text { trabajo }\end{array}$ & $\begin{array}{c}\text { HOMBRES } \\
(\mathrm{N}=80) \\
\text { MUJERES } \\
(\mathrm{N}=125)\end{array}$ & $\begin{array}{l}4.28 \\
3.97\end{array}$ & $\begin{array}{l}1.16 \\
1.20\end{array}$ & .004 & .952 & -1.852 & 203 & .065 \\
\hline $\begin{array}{l}\text { Compromiso } \\
\text { con el trabajo }\end{array}$ & $\begin{array}{l}\text { HOMBRES } \\
(\mathrm{N}=80) \\
\text { MUJERES } \\
(\mathrm{N}=125)\end{array}$ & $\begin{array}{l}5.80 \\
5.95\end{array}$ & $\begin{array}{l}0.69 \\
0.58\end{array}$ & 1.083 & .299 & 1.690 & 203 & .093 \\
\hline $\begin{array}{l}\text { Compromiso de } \\
\text { conveniencia }\end{array}$ & $\begin{array}{c}\text { HOMBRES } \\
(\mathrm{N}=80) \\
\text { MUJERES } \\
(\mathrm{N}=125)\end{array}$ & $\begin{array}{l}3.61 \\
3.61\end{array}$ & $\begin{array}{l}0.75 \\
0.92\end{array}$ & 4.172 & .042 & .037 & 192 & .970 \\
\hline $\begin{array}{l}\text { Compromiso } \\
\text { afectivo }\end{array}$ & $\begin{array}{c}\text { HOMBRES } \\
(\mathrm{N}=80) \\
\text { MUJERES } \\
(\mathrm{N}=125)\end{array}$ & $\begin{array}{l}4.55 \\
4.54\end{array}$ & $\begin{array}{l}0.68 \\
0.61\end{array}$ & .577 & .448 & -.051 & 203 & .959 \\
\hline $\begin{array}{l}\text { Sentimiento de } \\
\text { permanencia }\end{array}$ & $\begin{array}{c}\text { HOMBRES } \\
(\mathrm{N}=80) \\
\text { MUJERES } \\
(\mathrm{N}=125)\end{array}$ & $\begin{array}{l}4.51 \\
4.59\end{array}$ & $\begin{array}{l}0.94 \\
0.92\end{array}$ & .120 & .729 & .638 & 203 & .524 \\
\hline $\begin{array}{l}\text { Jerarquía del } \\
\text { puesto }\end{array}$ & $\begin{array}{c}\text { HOMBRES } \\
(\mathrm{N}=80) \\
\text { MUJERES } \\
(\mathrm{N}=125)\end{array}$ & $\begin{array}{l}4.84 \\
4.69\end{array}$ & $\begin{array}{l}0.82 \\
1.12\end{array}$ & 5.744 & .017 & -1.143 & 199 & .254 \\
\hline
\end{tabular}


Cuadro 7. Comparación de factores de compromiso organizacional por condiciones laborales.

\begin{tabular}{|c|c|c|c|c|c|c|c|c|}
\hline \multirow[t]{2}{*}{ VARIABLES } & \multirow[b]{2}{*}{ Cond. Labor } & \multirow[b]{2}{*}{$\mathbf{M}$} & \multirow[b]{2}{*}{ D. $\mathbf{S}$. } & \multicolumn{2}{|c|}{ Prueba de Varianza } & \multicolumn{3}{|c|}{ Prueba de Medias } \\
\hline & & & & $\mathbf{F}$ & $\mathbf{P}$ & t & gl & $\mathbf{p}$ \\
\hline $\begin{array}{l}\text { Satisfacción } \\
\text { general en el } \\
\text { trabajo }\end{array}$ & \begin{tabular}{|c|} 
Contratado \\
$(\mathrm{N}=103)$ \\
Nombrado \\
$(\mathrm{N}=102)$
\end{tabular} & $\begin{array}{l}4.10 \\
4.08\end{array}$ & $\begin{array}{l}1.11 \\
1.27\end{array}$ & 1.67 & 0.20 & .127 & 203 & .899 \\
\hline $\begin{array}{l}\text { Compromiso } \\
\text { con el trabajo }\end{array}$ & $\begin{array}{c}\text { Contratado } \\
(\mathrm{N}=103) \\
\text { Nombrado } \\
(\mathrm{N}=102)\end{array}$ & $\begin{array}{l}5.92 \\
5.86\end{array}$ & $\begin{array}{l}0.61 \\
0.65\end{array}$ & .734 & .393 & .638 & 203 & .524 \\
\hline $\begin{array}{l}\text { Compromiso de } \\
\text { conveniencia }\end{array}$ & $\begin{array}{c}\text { Contratado } \\
(\mathrm{N}=103) \\
\text { Nombrado } \\
(\mathrm{N}=102)\end{array}$ & $\begin{array}{l}3,58 \\
3.64\end{array}$ & $\begin{array}{l}0.82 \\
0.89\end{array}$ & 2.328 & 0.129 & -.487 & 203 & .627 \\
\hline $\begin{array}{l}\text { Compromiso } \\
\text { afectivo }\end{array}$ & $\begin{array}{l}\text { Contratado } \\
(\mathrm{N}=103) \\
\text { Nombrado } \\
(\mathrm{N}=102)\end{array}$ & $\begin{array}{l}4.50 \\
4.59\end{array}$ & $\begin{array}{l}0.62 \\
0.64\end{array}$ & .130 & .719 & -1.088 & 203 & .278 \\
\hline $\begin{array}{l}\text { Sentimiento de } \\
\text { permanencia }\end{array}$ & $\begin{array}{c}\text { Contratado } \\
(\mathrm{N}=103) \\
\text { Nombrado } \\
(\mathrm{N}=102) \\
\end{array}$ & $\begin{array}{l}4.65 \\
4.48\end{array}$ & $\begin{array}{l}0.89 \\
0.96\end{array}$ & 2.496 & .116 & 1.318 & 203 & .189 \\
\hline $\begin{array}{l}\text { Jerarquía del } \\
\text { puesto }\end{array}$ & $\begin{array}{c}\text { Contratado } \\
(\mathrm{N}=103) \\
\text { Nombrado } \\
(\mathrm{N}=102)\end{array}$ & $\begin{array}{l}4.74 \\
4.76\end{array}$ & $\begin{array}{l}0.94 \\
1.09\end{array}$ & 1.276 & .260 & -.093 & 203 & .926 \\
\hline
\end{tabular}

Cuadro 8. Comparación de factores de compromiso organizacional por grupos de edad.

\begin{tabular}{|c|c|c|c|c|c|c|c|c|c|}
\hline \multicolumn{4}{|l|}{ VARIABLES } & \multicolumn{4}{|c|}{ Prueba de Varianza } & \multicolumn{2}{|c|}{ Anova } \\
\hline & Grupos de edad & M & D. $\mathbf{S}$. & Levene & gl 1 & gl 2 & p & $\mathbf{F}$ & $\mathbf{P}$ \\
\hline Satisfacción & $21-30(\mathrm{n}: 54)$ & 4.10 & 1.10 & \multirow{4}{*}{.228} & \multirow{4}{*}{3} & \multirow{4}{*}{201} & \multirow{4}{*}{.877} & \multirow{4}{*}{2.396} & \multirow{4}{*}{.069} \\
\hline general en el & 31-40 (n:64) & 3.90 & 1.15 & & & & & & \\
\hline trabajo & $41-50(n: 58)$ & 4.04 & 1.26 & & & & & & \\
\hline & $51-60(n: 29)$ & 4.60 & 1.21 & & & & & & \\
\hline \multirow{4}{*}{$\begin{array}{l}\text { Compromiso } \\
\text { con el trabajo }\end{array}$} & $21-30$ (n:54) & 5.86 & 0.57 & \multirow{4}{*}{.194} & \multirow{4}{*}{3} & \multirow{4}{*}{201} & \multirow{4}{*}{.901} & \multirow{4}{*}{.336} & \multirow{4}{*}{.799} \\
\hline & $31-40(\mathrm{n}: 64)$ & 5.94 & 0.68 & & & & & & \\
\hline & $41-50(n: 58)$ & 5.90 & 0.65 & & & & & & \\
\hline & $51-60(\mathrm{n}: 29)$ & 5.81 & 0.57 & & & & & & \\
\hline \multirow{4}{*}{$\begin{array}{l}\text { Compromiso de } \\
\text { conveniencia }\end{array}$} & $21-30(\mathrm{n}: 54)$ & 3.58 & 0.87 & \multirow{4}{*}{.460} & \multirow{4}{*}{3} & \multirow{4}{*}{201} & \multirow{4}{*}{.710} & \multirow{4}{*}{.098} & \multirow{4}{*}{.961} \\
\hline & $31-40(n: 64)$ & 3.59 & 0.81 & & & & & & \\
\hline & $41-50$ (n:58) & 3.66 & 0.88 & & & & & & \\
\hline & $51-60(n: 29)$ & 3.63 & 0.93 & & & & & & \\
\hline \multirow{4}{*}{$\begin{array}{l}\text { Compromiso } \\
\text { afectivo }\end{array}$} & $21-30(\mathrm{n}: 54)$ & 4.51 & 0.61 & \multirow{4}{*}{.237} & \multirow{4}{*}{3} & \multirow{4}{*}{201} & \multirow{4}{*}{.871} & \multirow{4}{*}{.477} & \multirow{4}{*}{.699} \\
\hline & $31-40(n: 64)$ & 4.49 & 0.66 & & & & & & \\
\hline & $41-50(n: 58)$ & 4.61 & 0.65 & & & & & & \\
\hline & $51-60$ (n:29) & 4.59 & 0.59 & & & & & & \\
\hline Sentimiento de & $21-30$ (n:54) & 4.67 & 1.03 & & & & & & \\
\hline permanencia & $31-40(n: 64)$ & 4.69 & 0.88 & & & & & & \\
\hline & $41-50(n: 58)$ & 4.43 & 0.91 & .222 & 3 & 201 & .881 & 1.512 & .212 \\
\hline & $51-60(\mathrm{n}: 29)$ & 4.35 & 0.83 & & & & & & \\
\hline Jerarquía del & $21-30$ (n:54) & 4.74 & 1.02 & & & & & & \\
\hline puesto & $31-40(\mathrm{n}: 64)$ & 4.83 & 1.01 & & & & & & \\
\hline & $41-50(n: 58)$ & 4.68 & 1.09 & .168 & 3 & 201 & .918 & 222 & .881 \\
\hline & $51-60(\mathrm{n}: 29)$ & 4.73 & 0.89 & & & & & & \\
\hline
\end{tabular}


Cuadro 9. Comparación de factores de compromiso organizacional por estado civil.

\begin{tabular}{|c|c|c|c|c|c|c|c|c|c|}
\hline \multicolumn{4}{|l|}{ VARIABLES } & \multicolumn{4}{|c|}{ Prueba de varianza } & \multicolumn{2}{|c|}{ Anova } \\
\hline & Estado civil & M & D. S. & Levene & gl 1 & gl 2 & $\mathbf{P}$ & $\mathbf{F}$ & $\mathbf{P}$ \\
\hline $\begin{array}{l}\text { Satisfacción } \\
\text { general en el } \\
\text { trabajo }\end{array}$ & $\begin{array}{r}\text { Soltero } \\
\text { Casado } \\
\text { Viudo } \\
\text { Divorciado } \\
\text { Unión libre }\end{array}$ & $\begin{array}{l}4.17 \\
4.08 \\
3.38 \\
4.20 \\
4.05\end{array}$ & $\begin{array}{l}1.12 \\
1.24 \\
1.35 \\
0.57 \\
1.33\end{array}$ & 1.968 & 4 & 200 & .101 & .722 & .578 \\
\hline $\begin{array}{l}\text { Compromiso } \\
\text { con el trabajo }\end{array}$ & $\begin{array}{r}\text { Soltero } \\
\text { Casado } \\
\text { Viudo } \\
\text { Divorciado } \\
\text { Unión libre }\end{array}$ & $\begin{array}{l}5.90 \\
5.88 \\
5.65 \\
6.00 \\
5.93\end{array}$ & $\begin{array}{l}0.58 \\
0.69 \\
0.50 \\
0.55 \\
0.54\end{array}$ & 1.220 & 4 & 200 & .304 & .342 & .849 \\
\hline $\begin{array}{l}\text { Compromiso de } \\
\text { conveniencia }\end{array}$ & $\begin{array}{r}\text { Soltero } \\
\text { Casado } \\
\text { Viudo } \\
\text { Divorciado } \\
\text { Unión libre }\end{array}$ & $\begin{array}{l}3.50 \\
3.75 \\
3.61 \\
3.21 \\
3.37\end{array}$ & $\begin{array}{l}0.80 \\
0.89 \\
0.74 \\
0.86 \\
0.83\end{array}$ & .345 & 4 & 200 & .847 & 1.698 & .152 \\
\hline $\begin{array}{l}\text { Compromiso } \\
\text { afectivo }\end{array}$ & $\begin{array}{r}\text { Soltero } \\
\text { Casado } \\
\text { Viudo } \\
\text { Divorciado } \\
\text { Unión libre }\end{array}$ & $\begin{array}{l}4.49 \\
4.59 \\
4.60 \\
4.46 \\
4.51\end{array}$ & $\begin{array}{l}0.64 \\
0.65 \\
0.57 \\
0.52 \\
0.64\end{array}$ & .377 & 4 & 200 & .825 & .334 & .855 \\
\hline $\begin{array}{l}\text { Sentimiento de } \\
\text { permanencia }\end{array}$ & $\begin{array}{r}\text { Soltero } \\
\text { Casado } \\
\text { Viudo } \\
\text { Divorciado } \\
\text { Unión libre }\end{array}$ & $\begin{array}{l}4.74 \\
4.52 \\
4.07 \\
4.16 \\
4.33\end{array}$ & $\begin{array}{l}0.94 \\
0.89 \\
0.72 \\
1.00 \\
1.01\end{array}$ & .408 & 4 & 200 & .803 & 1.867 & .118 \\
\hline $\begin{array}{l}\text { Jerarquía del } \\
\text { puesto }\end{array}$ & $\begin{array}{r}\text { Soltero } \\
\text { Casado } \\
\text { Viudo } \\
\text { Divorciado } \\
\text { Unión libre }\end{array}$ & $\begin{array}{l}4.80 \\
4.66 \\
4.91 \\
5.30 \\
4.77\end{array}$ & $\begin{array}{l}0.96 \\
1.08 \\
1.09 \\
0.70 \\
0.89\end{array}$ & .527 & 4 & 200 & .716 & .889 & .471 \\
\hline
\end{tabular}

$\begin{array}{ll}\text { Soltero: } & (N=77) \\ \text { Casado: } & (N=100) \\ \text { Viudo: } & (N=7) \\ \text { Divorciado: } & (N=8) \\ \text { Unión libre: } & (N=13)\end{array}$


Cuadro 10. Comparación de los factores de compromiso organizacional por nivel máximo de estudios.

\begin{tabular}{|c|c|c|c|c|c|c|c|c|c|}
\hline \multirow[t]{2}{*}{ VARIABLES } & \multirow[b]{2}{*}{ Nivel estudio } & \multirow[b]{2}{*}{$\mathbf{M}$} & \multirow[b]{2}{*}{ D. S. } & \multicolumn{4}{|c|}{ PRUEBA DE VARIANZA } & \multicolumn{2}{|c|}{ ANOVA } \\
\hline & & & & Levene & gl 1 & gl 2 & $\mathbf{p}$ & $\mathbf{F}$ & $\mathbf{P}$ \\
\hline $\begin{array}{l}\text { Satisfacción } \\
\text { general en el } \\
\text { trabajo }\end{array}$ & $\begin{array}{r}\text { Secundaria } \\
\text { Técnico } \\
\text { Superior in } \\
\text { Superior co } \\
\text { Profesional } \\
\text { Pos grado } \\
\end{array}$ & $\begin{array}{l}4.41 \\
4.04 \\
4.24 \\
4.11 \\
3.55 \\
3.90 \\
\end{array}$ & $\begin{array}{r}.72 \\
1.25 \\
1.20 \\
1.05 \\
1.45 \\
1.76 \\
\end{array}$ & 2.888 & 5 & 199 & .015 & 1.540 & .179 \\
\hline $\begin{array}{l}\text { Compromiso } \\
\text { con el trabajo }\end{array}$ & $\begin{array}{r}\text { Secundaria } \\
\text { Técnico } \\
\text { Superior in } \\
\text { Superior co } \\
\text { Profesional } \\
\text { Pos grado }\end{array}$ & $\begin{array}{l}5.64 \\
5.80 \\
6.00 \\
6.08 \\
5.90 \\
5.99\end{array}$ & $\begin{array}{l}.68 \\
.69 \\
.50 \\
.56 \\
.64 \\
.42\end{array}$ & 1.239 & 5 & 199 & .292 & 2.358 & .042 \\
\hline $\begin{array}{l}\text { Compromiso de } \\
\text { conveniencia }\end{array}$ & $\begin{array}{r}\text { Secundaria } \\
\text { Técnico } \\
\text { Superior in } \\
\text { Superior co } \\
\text { Profesional } \\
\text { Pos grado }\end{array}$ & $\begin{array}{l}3.73 \\
3.77 \\
3.50 \\
3.57 \\
3.46 \\
3.00\end{array}$ & $\begin{array}{l}.58 \\
.83 \\
.87 \\
.95 \\
.92 \\
.98\end{array}$ & 1.402 & 5 & 199 & .225 & 1.480 & .198 \\
\hline $\begin{array}{l}\text { Compromiso } \\
\text { afectivo }\end{array}$ & $\begin{array}{r}\text { Secundaria } \\
\text { Técnico } \\
\text { Superior in } \\
\text { Superior co } \\
\text { Profesional } \\
\text { Pos grado }\end{array}$ & $\begin{array}{l}4.56 \\
4.58 \\
4.46 \\
4.54 \\
4.63 \\
4.40\end{array}$ & $\begin{array}{l}.53 \\
.69 \\
.58 \\
.67 \\
.60 \\
.85\end{array}$ & 1.121 & 5 & 199 & .350 & .337 & .890 \\
\hline $\begin{array}{l}\text { Sentimiento de } \\
\text { permanencia }\end{array}$ & $\begin{array}{r}\text { Secundaria } \\
\text { Técnico } \\
\text { Superior in } \\
\text { Superior co } \\
\text { Profesional } \\
\text { Pos grado }\end{array}$ & $\begin{array}{l}4.22 \\
4.57 \\
4.39 \\
4.77 \\
4.63 \\
5.54\end{array}$ & $\begin{array}{r}1.13 \\
.97 \\
.78 \\
.80 \\
.78 \\
.85\end{array}$ & 1.501 & 5 & 199 & .191 & 2.987 & .013 \\
\hline $\begin{array}{l}\text { Jerarquía del } \\
\text { puesto }\end{array}$ & $\begin{array}{r}\text { Secundaria } \\
\text { Técnico } \\
\text { Superior in } \\
\text { Superior co } \\
\text { Profesional } \\
\text { Pos grado }\end{array}$ & $\begin{array}{l}4.56 \\
4.82 \\
4.73 \\
4.93 \\
4.45 \\
4.87\end{array}$ & $\begin{array}{r}.84 \\
1.09 \\
.84 \\
.98 \\
1.13 \\
1.78\end{array}$ & 1.702 & 5 & 199 & .136 & .919 & .470 \\
\hline $\begin{array}{l}\text { Secundaria: } \\
\text { Técnico: } \\
\text { Superior incompleta: } \\
\text { Superior completa: } \\
\text { Profesional: } \\
\text { Postgrado: }\end{array}$ & $\begin{array}{l}(\mathrm{N}=29) \\
(\mathrm{N}=65) \\
(\mathrm{N}=40) \\
(\mathrm{N}=43) \\
(\mathrm{N}=22) \\
(\mathrm{N}=6)\end{array}$ & & & & & & & & \\
\hline
\end{tabular}


Cuadro 11. Comparación de los factores de compromiso organizacional por número de trabajadores.

\begin{tabular}{|c|c|c|c|c|c|c|c|c|c|}
\hline \multicolumn{4}{|l|}{ VARIABLES } & \multicolumn{4}{|c|}{ Prueba de varianza } & \multicolumn{2}{|c|}{ Anova } \\
\hline & $\mathbf{N}^{0}$ Trabajadores & $\mathbf{M}$ & D. S. & Levene & gl 1 & gl 2 & $\mathbf{p}$ & $\mathbf{F}$ & $\mathbf{P}$ \\
\hline $\begin{array}{l}\text { Satisfacción } \\
\text { general en el } \\
\text { trabajo }\end{array}$ & $\begin{array}{r}-25(\mathrm{n}: 5) \\
26-50(\mathrm{n}: 126) \\
51-100(\mathrm{n}: 43) \\
101-200(\mathrm{n}: 16) \\
201-500(\mathrm{n}: 15)\end{array}$ & $\begin{array}{l}3.95 \\
4.06 \\
4.22 \\
3.82 \\
4.09\end{array}$ & $\begin{array}{r}.70 \\
1.18 \\
1.32 \\
1.22 \\
.94\end{array}$ & 1.220 & 4 & 200 & .304 & .467 & .760 \\
\hline $\begin{array}{l}\text { Compromiso con } \\
\text { el trabajo }\end{array}$ & $\begin{array}{r}-25(\mathrm{n}: 5) \\
26-50(\mathrm{n}: 126) \\
51-100(\mathrm{n}: 43) \\
101-200(\mathrm{n}: 16) \\
201-500(\mathrm{n}: 15)\end{array}$ & $\begin{array}{l}6.16 \\
5.91 \\
5.89 \\
5.86 \\
5.64\end{array}$ & $\begin{array}{l}.51 \\
.59 \\
.58 \\
.77 \\
.89 \\
\end{array}$ & .997 & 4 & 200 & .411 & .870 & .483 \\
\hline $\begin{array}{l}\text { Compromiso de } \\
\text { conveniencia }\end{array}$ & $\begin{array}{r}-25(\mathrm{n}: 5) \\
26-50(\mathrm{n}: 126) \\
51-100(\mathrm{n}: 43) \\
101-200(\mathrm{n}: 16) \\
201-500(\mathrm{n}: 15) \\
\end{array}$ & $\begin{array}{l}3.87 \\
3.66 \\
3.55 \\
3.23 \\
3.70 \\
\end{array}$ & $\begin{array}{l}.38 \\
.85 \\
.91 \\
.83 \\
.83 \\
\end{array}$ & .964 & 4 & 200 & .428 & 1.093 & .361 \\
\hline $\begin{array}{l}\text { Compromiso } \\
\text { afectivo }\end{array}$ & $\begin{array}{r}-25(\mathrm{n}: 5) \\
26-50(\mathrm{n}: 126) \\
51-100(\mathrm{n}: 43) \\
101-200(\mathrm{n}: 16) \\
201-500(\mathrm{n}: 15)\end{array}$ & $\begin{array}{l}4.73 \\
4.60 \\
4.51 \\
4.39 \\
4.27\end{array}$ & $\begin{array}{l}.72 \\
.63 \\
.63 \\
.67 \\
.62\end{array}$ & .124 & 4 & 200 & .974 & 1.360 & .249 \\
\hline $\begin{array}{l}\text { Sentimiento de } \\
\text { permanencia }\end{array}$ & $\begin{array}{r}-25(\mathrm{n}: 5) \\
26-50(\mathrm{n}: 126) \\
51-100(\mathrm{n}: 43) \\
101-200(\mathrm{n}: 16) \\
201-500(\mathrm{n}: 15)\end{array}$ & $\begin{array}{l}5.00 \\
4.68 \\
4.39 \\
4.34 \\
4.18\end{array}$ & $\begin{array}{r}1.01 \\
.88 \\
.99 \\
1.04 \\
.82\end{array}$ & .332 & 4 & 200 & .856 & 2.035 & .091 \\
\hline $\begin{array}{l}\text { Jerarquía del } \\
\text { puesto }\end{array}$ & $\begin{array}{r}-25(\mathrm{n}: 5) \\
26-50(\mathrm{n}: 126) \\
51-100(\mathrm{n}: 43) \\
101-200(\mathrm{n}: 16) \\
201-500(\mathrm{n}: 15)\end{array}$ & $\begin{array}{l}5.04 \\
4.67 \\
4.80 \\
4.90 \\
5.01\end{array}$ & $\begin{array}{r}.86 \\
1.05 \\
.95 \\
1.19 \\
.75\end{array}$ & .585 & 4 & 200 & .674 & .640 & .634 \\
\hline
\end{tabular}


Cuadro 12. Comparacion de los factores de compromiso organizacional por antigüedad en la universidad.

\begin{tabular}{|c|c|c|c|c|c|c|c|c|c|}
\hline \multicolumn{4}{|l|}{ VARIABLES } & \multicolumn{4}{|c|}{ Prueba de varianza } & \multicolumn{2}{|c|}{ Anova } \\
\hline & ntigüedad $U$. & M & D. $S$. & Levene & gl 1 & gl 2 & $\mathbf{p}$ & $\mathbf{F}$ & $\mathbf{P}$ \\
\hline $\begin{array}{l}\text { Satisfacción } \\
\text { general en el } \\
\text { trabajo }\end{array}$ & $\begin{array}{r}-1(\mathrm{n}: 11) \\
1-5(\mathrm{n}: 52) \\
6-10(\mathrm{n}: 32) \\
11-20(\mathrm{n}: 64) \\
21-30(\mathrm{n}: 41) \\
+30(\mathrm{n}: 5)\end{array}$ & $\begin{array}{l}4.56 \\
3.96 \\
4.05 \\
4.05 \\
4.13 \\
4.81\end{array}$ & $\begin{array}{c}.98 \\
1.14 \\
1.18 \\
1.30 \\
1.12 \\
1.28\end{array}$ & .584 & 5 & 199 & .712 & .857 & .511 \\
\hline $\begin{array}{l}\text { Compromiso } \\
\text { con el trabajo }\end{array}$ & $\begin{array}{r}-1(\mathrm{n}: 11) \\
1-5(\mathrm{n}: 52) \\
6-10(\mathrm{n}: 32) \\
11-20(\mathrm{n}: 64) \\
21-30(\mathrm{n}: 41) \\
+30(\mathrm{n}: 5)\end{array}$ & $\begin{array}{l}5.85 \\
5.88 \\
6.02 \\
5.79 \\
5.97 \\
5.96\end{array}$ & $\begin{array}{l}.51 \\
.64 \\
.48 \\
.76 \\
.51 \\
.68\end{array}$ & 1.815 & 5 & 199 & .111 & .787 & .560 \\
\hline $\begin{array}{l}\text { Compromiso de } \\
\text { conveniencia }\end{array}$ & $\begin{array}{r}-1(\mathrm{n}: 11) \\
1-5(\mathrm{n}: 52) \\
6-10(\mathrm{n}: 32) \\
11-20(\mathrm{n}: 64) \\
21-30(\mathrm{n}: 41) \\
+30(\mathrm{n}: 5)\end{array}$ & $\begin{array}{l}3.07 \\
3.73 \\
3.51 \\
3.79 \\
3.46 \\
3.18 \\
\end{array}$ & $\begin{array}{l}.64 \\
.79 \\
.84 \\
.85 \\
.88 \\
.93\end{array}$ & .591 & 5 & 199 & .707 & .2 .339 & .043 \\
\hline $\begin{array}{l}\text { Compromiso } \\
\text { afectivo }\end{array}$ & $\begin{array}{r}-1(\mathrm{n}: 11) \\
1-5(\mathrm{n}: 52) \\
6-10(\mathrm{n}: 32) \\
11-20(\mathrm{n}: 64) \\
21-30(\mathrm{n}: 41) \\
+30(\mathrm{n}: 5) \\
\end{array}$ & $\begin{array}{l}4.31 \\
4.44 \\
4.62 \\
4.69 \\
4.47 \\
4.41 \\
\end{array}$ & $\begin{array}{l}.49 \\
.73 \\
.56 \\
.62 \\
.60 \\
.50 \\
\end{array}$ & 1.153 & 5 & 199 & .334 & 1.518 & .186 \\
\hline $\begin{array}{l}\text { Sentimiento de } \\
\text { permanencia }\end{array}$ & $\begin{array}{r}-1(\mathrm{n}: 11) \\
1-5(\mathrm{n}: 52) \\
6-10(\mathrm{n}: 32) \\
11-20(\mathrm{n}: 64) \\
21-30(\mathrm{n}: 41) \\
+30(\mathrm{n}: 5)\end{array}$ & $\begin{array}{l}4.49 \\
4.67 \\
4.71 \\
4.61 \\
4.27 \\
4.28\end{array}$ & $\begin{array}{c}1.19 \\
.71 \\
1.18 \\
.96 \\
.80 \\
.68\end{array}$ & 2.502 & 5 & 199 & .032 & $1-292$ & .269 \\
\hline $\begin{array}{l}\text { Jerarquía del } \\
\text { puesto }\end{array}$ & $\begin{array}{r}-1 \\
(\mathrm{n}: 11) \\
1-5 \\
(\mathrm{n}: 52) \\
6-10 \\
(\mathrm{n}: 32) \\
11-20 \\
(\mathrm{n}: 64) \\
21-30 \\
(\mathrm{n}: 41) \\
+30 \\
(\mathrm{n}: 5)\end{array}$ & $\begin{array}{l}4.71 \\
4.84 \\
4.61 \\
4.68 \\
4.83 \\
4.92\end{array}$ & $\begin{array}{c}.77 \\
.96 \\
1.08 \\
1.14 \\
.93 \\
.78\end{array}$ & .569 & 5 & 199 & .708 & .340 & .888 \\
\hline
\end{tabular}


Cuadro 13. Comparación de los factores de compromiso organizacional por antigüedad en el puesto actual.

\begin{tabular}{|c|c|c|c|c|c|c|c|c|c|c|}
\hline \multirow{2}{*}{\multicolumn{3}{|c|}{ Antig. puesto actu }} & \multirow[b]{2}{*}{$\mathbf{M}$} & \multirow{2}{*}{$\begin{array}{l}\text { D. S. } \\
1.26 \\
1.16\end{array}$} & \multicolumn{4}{|c|}{ Prueba de varianza } & \multicolumn{2}{|c|}{ Anova } \\
\hline & & & & & Levene & gl 1 & gl 2 & $\mathbf{p}$ & $\mathbf{F}$ & $\mathbf{P}$ \\
\hline $\begin{array}{l}\text { Satisfacción } \\
\text { general en el } \\
\text { trabajo }\end{array}$ & $\begin{array}{l}-1 \\
(\mathrm{n}: 58) \\
1-5 \\
(\mathrm{n}: 96) \\
6-10 \\
(\mathrm{n}: 24)\end{array}$ & $\begin{array}{l}11-20 \\
(\mathrm{n}: 21) \\
21-30 \\
(\mathrm{n}: 5) \\
+30 \\
(\mathrm{n}: 1)\end{array}$ & $\begin{array}{l}4.18 \\
3.98 \\
4.23 \\
4.24 \\
3.63 \\
5.67 \\
\end{array}$ & $\begin{array}{c}1.26 \\
1.16 \\
1.15 \\
1.19 \\
.92 \\
---\end{array}$ & .551 & 4 & 199 & .698 & .873 & .500 \\
\hline $\begin{array}{l}\text { Compromiso } \\
\text { con el trabajo }\end{array}$ & $\begin{array}{l}-1 \\
(\mathrm{n}: 58) \\
1-5 \\
(\mathrm{n}: 96) \\
6-10 \\
(\mathrm{n}: 24)\end{array}$ & $\begin{array}{l}11-20 \\
(\mathrm{n}: 21) \\
21-30 \\
(\mathrm{n}: 5) \\
+30 \\
(\mathrm{n}: 1)\end{array}$ & $\begin{array}{l}5.94 \\
5.89 \\
6.04 \\
5.65 \\
5.80 \\
5.37 \\
\end{array}$ & $\begin{array}{l}.53 \\
.66 \\
.54 \\
.76 \\
.75 \\
--- \\
\end{array}$ & .955 & 4 & 199 & .433 & 1.081 & .372 \\
\hline $\begin{array}{l}\text { Compromiso de } \\
\text { Conveniencia }\end{array}$ & $\begin{array}{l}-1 \\
(\mathrm{n}: 58) \\
1-5 \\
(\mathrm{n}: 96) \\
6-10 \\
(\mathrm{n}: 24)\end{array}$ & $\begin{array}{l}11-20 \\
(\mathrm{n}: 21) \\
21-30 \\
(\mathrm{n}: 5) \\
+30 \\
(\mathrm{n}: 1)\end{array}$ & $\begin{array}{l}3.52 \\
3.68 \\
3.21 \\
3.89 \\
4.22 \\
3.18 \\
\end{array}$ & $\begin{array}{l}.76 \\
.90 \\
.80 \\
.82 \\
.70 \\
--- \\
\end{array}$ & .987 & 4 & 199 & .416 & 2.400 & .039 \\
\hline $\begin{array}{l}\text { Compromiso } \\
\text { afectivo }\end{array}$ & $\begin{array}{l}-1 \\
(\mathrm{n}: 58) \\
1-5 \\
(\mathrm{n}: 96) \\
6-10 \\
(\mathrm{n}: 24)\end{array}$ & $\begin{array}{l}11-20 \\
(\mathrm{n}: 21) \\
21-30 \\
(\mathrm{n}: 5) \\
+30 \\
(\mathrm{n}: 1)\end{array}$ & $\begin{array}{l}4.51 \\
4.50 \\
4.63 \\
4.71 \\
4.79 \\
4.19 \\
\end{array}$ & $\begin{array}{l}.62 \\
.67 \\
.57 \\
.61 \\
.51 \\
--- \\
\end{array}$ & .421 & 4 & 199 & .793 & .742 & .593 \\
\hline $\begin{array}{l}\text { Sentimiento de } \\
\text { permanencia }\end{array}$ & $\begin{array}{l}-1 \\
(\mathrm{n}: 58) \\
1-5 \\
(\mathrm{n}: 96) \\
6-10 \\
(\mathrm{n}: 24)\end{array}$ & $\begin{array}{l}11-20 \\
(\mathrm{n}: 21) \\
21-30 \\
(\mathrm{n}: 5) \\
+30 \\
(\mathrm{n}: 1)\end{array}$ & $\begin{array}{l}4.68 \\
4.51 \\
4.59 \\
4.62 \\
3.88 \\
4.50 \\
\end{array}$ & $\begin{array}{c}.90 \\
.86 \\
1.16 \\
1.07 \\
.56 \\
--- \\
\end{array}$ & 2.289 & 4 & 199 & .061 & .833 & .528 \\
\hline $\begin{array}{l}\text { Jerarquía del } \\
\text { puesto }\end{array}$ & $\begin{array}{l}-1 \\
(\mathrm{n}: 58) \\
1-5 \\
(\mathrm{n}: 96) \\
6-10 \\
(\mathrm{n}: 24)\end{array}$ & $\begin{array}{l}11-20 \\
(\mathrm{n}: 21) \\
21-30 \\
(\mathrm{n}: 5) \\
+30 \\
(\mathrm{n}: 1)\end{array}$ & $\begin{array}{l}4.80 \\
4.77 \\
4.44 \\
4.75 \\
5.04 \\
5.80\end{array}$ & $\begin{array}{c}1.00 \\
.97 \\
1.09 \\
1.20 \\
.93 \\
---\end{array}$ & .261 & 4 & 199 & .903 & .774 & .570 \\
\hline
\end{tabular}


Cuadro 14. Comparación de los factores de compromiso organizacional por puesto actual.

\begin{tabular}{|c|c|c|c|c|c|c|c|c|c|}
\hline \multirow[t]{2}{*}{ VARIABLES } & \multirow[b]{2}{*}{ Puesto actual } & & & \multicolumn{4}{|c|}{ Prueba de varianza } & \multicolumn{2}{|c|}{ Anova } \\
\hline & & $\mathbf{M}$ & D. S. & Levene & gl 1 & gl 2 & $\mathbf{p}$ & $\mathbf{F}$ & $\mathbf{P}$ \\
\hline \multirow{6}{*}{$\begin{array}{l}\text { Satisfacción } \\
\text { general en el } \\
\text { trabajo }\end{array}$} & Director o jefe & 3.63 & 1.36 & \multirow{6}{*}{.611} & \multirow{6}{*}{5} & \multirow{6}{*}{199} & \multirow{6}{*}{.691} & \multirow{6}{*}{.598} & \multirow{6}{*}{.702} \\
\hline & Funcionario II & 4.23 & 1.18 & & & & & & \\
\hline & Funcionario I & 4.13 & 1.51 & & & & & & \\
\hline & Empleado & 4.07 & 1.14 & & & & & & \\
\hline & Trabajo manual & 4.21 & 1.17 & & & & & & \\
\hline & Otros & 3.68 & 1.34 & & & & & & \\
\hline \multirow{6}{*}{$\begin{array}{l}\text { Compromiso } \\
\text { con el trabajo }\end{array}$} & Director o jefe & 6.16 & .89 & \multirow{6}{*}{1.042} & \multirow{6}{*}{5} & \multirow{6}{*}{199} & \multirow{6}{*}{.394} & \multirow{6}{*}{1.185} & \multirow{6}{*}{.318} \\
\hline & Funcionario II & 5.93 & .57 & & & & & & \\
\hline & Funcionario I & 5.96 & .70 & & & & & & \\
\hline & Empleado & 5.93 & .58 & & & & & & \\
\hline & Trabajo manual & 5.60 & .76 & & & & & & \\
\hline & Otros & 5.80 & .82 & & & & & & \\
\hline \multirow{6}{*}{$\begin{array}{l}\text { Compromiso de } \\
\text { conveniencia }\end{array}$} & Director o jefe & 4.20 & .69 & \multirow{6}{*}{1.627} & \multirow{6}{*}{5} & \multirow{6}{*}{199} & \multirow{6}{*}{.154} & \multirow{6}{*}{2.287} & \multirow{6}{*}{.048} \\
\hline & Funcionario II & 3.34 & .99 & & & & & & \\
\hline & Funcionario I & 3.24 & .72 & & & & & & \\
\hline & Empleado & 3.69 & .83 & & & & & & \\
\hline & Trabajo manual & 3.73 & .65 & & & & & & \\
\hline & Otros & 3.89 & .75 & & & & & & \\
\hline & Director o jefe & 4.33 & .85 & & & & & & \\
\hline & Funcionario II & 4.55 & .60 & & & & & & \\
\hline Compromiso & Funcionario I & 4.80 & .65 & & & & & & \\
\hline afectivo & Empleado & 4.59 & .61 & .381 & 5 & 199 & .862 & 1.746 & .126 \\
\hline & Trabajo manual & 4.44 & .67 & & & & & & \\
\hline & Otros & 4.16 & .69 & & & & & & \\
\hline & Director o jefe & 5.42 & .80 & & & & & & \\
\hline & Funcionario II & 4.53 & .87 & & & & & & \\
\hline Sentimiento de & Funcionario I & 4.83 & .84 & & & & & & \\
\hline permanencia & Empleado & 4.56 & .87 & 1.344 & 5 & 199 & .247 & .896 & .485 \\
\hline & Trabajo manual & 4.42 & 1.17 & & & & & & \\
\hline & Otros & 4.42 & 1.23 & & & & & & \\
\hline & Director o jefe & 4.93 & 1.70 & & & & & & \\
\hline & Funcionario II & 4.93 & .98 & & & & & & \\
\hline Jerarquía del & Funcionario I & 4.89 & .83 & & & & & & \\
\hline puesto & Empleado & 4.75 & 1.04 & .574 & 5 & 199 & .720 & 1.00 & .414 \\
\hline & Trabajo manual & 4.35 & .94 & & & & & & \\
\hline & Otros & 4.66 & 1.05 & & & & & & \\
\hline
\end{tabular}


Cuadro 15. Comparación de unidades de trabajo (facultades) con factores de compromiso organizacional.

\begin{tabular}{|c|c|c|c|c|c|c|c|c|c|}
\hline \multirow[t]{2}{*}{ VARIABLES } & \multirow[b]{2}{*}{ Facultad } & \multirow[b]{2}{*}{$\mathbf{M}$} & \multirow[b]{2}{*}{ D. S. } & \multicolumn{4}{|c|}{ Prueba de varianza } & \multicolumn{2}{|c|}{ Anova } \\
\hline & & & & Levene & gl 1 & gl 2 & $\mathbf{p}$ & $\mathbf{F}$ & $\mathbf{P}$ \\
\hline $\begin{array}{l}\text { Satisfacción } \\
\text { general en el } \\
\text { trabajo }\end{array}$ & $\begin{array}{l}\text { Química } \\
\text { Matemáticas } \\
\text { Física } \\
\text { Biología } \\
\text { Veterinaria } \\
\text { CC. SS. } \\
\text { Administración } \\
\text { Contabilidad } \\
\text { Economía } \\
\text { Letras } \\
\text { Educación } \\
\text { Psicología } \\
\text { Electrónica } \\
\text { Ing. Industrial } \\
\text { Geología } \\
\text { Medicina } \\
\text { Farmacia } \\
\text { Ing. Sistemas } \\
\text { Odontología } \\
\text { Derecho }\end{array}$ & \begin{tabular}{l|l}
4.17 \\
4.28 \\
4.23 \\
3.86 \\
4.01 \\
4.70 \\
4.27 \\
3.93 \\
4.17 \\
4.58 \\
4.05 \\
3.36 \\
3.81 \\
3.96 \\
4.38 \\
3.73 \\
4.77 \\
3.55 \\
4.32 \\
3.85 \\
\end{tabular} & $\begin{array}{l}1.06 \\
.95 \\
1.16 \\
1.66 \\
.78 \\
1.05 \\
1.48 \\
1.33 \\
1.13 \\
1.03 \\
1.76 \\
.78 \\
1.10 \\
1.38 \\
.93 \\
.97 \\
1.36 \\
.90 \\
1.08 \\
1.40 \\
\end{array}$ & 1.489 & 19 & 185 & .093 & .949 & .523 \\
\hline $\begin{array}{l}\text { Compromiso con } \\
\text { el trabajo }\end{array}$ & $\begin{array}{l}\text { Química } \\
\text { Matemáticas } \\
\text { Física } \\
\text { Biología } \\
\text { Veterinaria } \\
\text { CC. SS. } \\
\text { Administración } \\
\text { Contabilidad } \\
\text { Economía } \\
\text { Letras } \\
\text { Educación } \\
\text { Psicología } \\
\text { Electrónica } \\
\text { Ing. Industrial } \\
\text { Geología } \\
\text { Medicina } \\
\text { Farmacia } \\
\text { Ing. Sistemas } \\
\text { Odontología } \\
\text { Derecho }\end{array}$ & $\begin{array}{l}5.64 \\
6.20 \\
5.67 \\
6.18 \\
5.55 \\
5.92 \\
6.38 \\
5.86 \\
5.77 \\
5.99 \\
6.07 \\
5.90 \\
5.96 \\
5.75 \\
5.97 \\
5.56 \\
5.91 \\
5.88 \\
5.90 \\
5.85\end{array}$ & $\begin{array}{l}.76 \\
.33 \\
.54 \\
.49 \\
.90 \\
.56 \\
.56 \\
.52 \\
.56 \\
.49 \\
.46 \\
.55 \\
.64 \\
.80 \\
.51 \\
.86 \\
.69 \\
.62 \\
.58 \\
.58\end{array}$ & 1.072 & 19 & 185 & .383 & 1.208 & .255 \\
\hline $\begin{array}{l}\text { Compromiso de } \\
\text { conveniencia }\end{array}$ & $\begin{array}{l}\text { Química } \\
\text { Matemáticas } \\
\text { Física } \\
\text { Biología } \\
\text { Veterinaria } \\
\text { CC. SS. } \\
\text { Administración } \\
\text { Contabilidad } \\
\text { Economía } \\
\text { Letras } \\
\text { Educación } \\
\text { Psicología } \\
\text { Electrónica } \\
\text { Ing. Industrial } \\
\text { Geología } \\
\text { Medicina } \\
\text { Farmacia } \\
\text { Ing. Sistemas } \\
\text { Odontología } \\
\text { Derecho }\end{array}$ & \begin{tabular}{l|}
3.91 \\
3.51 \\
3.68 \\
3.41 \\
3.21 \\
3.48 \\
3.52 \\
3.50 \\
3.35 \\
3.51 \\
3.45 \\
3.92 \\
3.45 \\
3.98 \\
4.16 \\
3.75 \\
3.30 \\
3.71 \\
3.72 \\
3.65 \\
\end{tabular} & $\begin{array}{l}.66 \\
.65 \\
.99 \\
.97 \\
.75 \\
.72 \\
.90 \\
.69 \\
.72 \\
1.08 \\
.89 \\
.87 \\
1.11 \\
.89 \\
.72 \\
.84 \\
.93 \\
.84 \\
.81 \\
.96 \\
\end{array}$ & .557 & 19 & 185 & .932 & .817 & .685 \\
\hline
\end{tabular}

Continúa.. 
...Viene de la p. 72

\begin{tabular}{|c|c|c|c|c|c|c|c|c|c|}
\hline $\begin{array}{l}\text { Compromiso } \\
\text { afectivo }\end{array}$ & $\begin{array}{l}\text { Química } \\
\text { Matemáticas } \\
\text { Física } \\
\text { Biología } \\
\text { Veterinaria } \\
\text { CC. SS. } \\
\text { Administración } \\
\text { Contabilidad } \\
\text { Economía } \\
\text { Letras } \\
\text { Educación } \\
\text { Psicología } \\
\text { Electrónica } \\
\text { Ing. Industrial } \\
\text { Geología } \\
\text { Medicina } \\
\text { Farmacia } \\
\text { Ing. Sistemas } \\
\text { Odontología } \\
\text { Derecho }\end{array}$ & $\begin{array}{l}4.66 \\
4.34 \\
4.59 \\
4.82 \\
4.21 \\
4.84 \\
4.98 \\
4.47 \\
4.68 \\
4.65 \\
4.57 \\
4.19 \\
4.53 \\
4.51 \\
4.71 \\
4.29 \\
4.54 \\
4.64 \\
4.41 \\
4.41\end{array}$ & $\begin{array}{l}.55 \\
.81 \\
.56 \\
.54 \\
.70 \\
.50 \\
.69 \\
.74 \\
.63 \\
.56 \\
.54 \\
.67 \\
.49 \\
.60 \\
.58 \\
.64 \\
.43 \\
.67 \\
.82 \\
.71\end{array}$ & .600 & 19 & 185 & .904 & 1.112 & .342 \\
\hline $\begin{array}{l}\text { Sentimiento de } \\
\text { permanencia }\end{array}$ & $\begin{array}{l}\text { Química } \\
\text { Matemáticas } \\
\text { Física } \\
\text { Biología }\end{array}$ & $\begin{array}{l}5.05 \\
4.69 \\
4.44 \\
4.55\end{array}$ & $\begin{array}{c}.86 \\
1.00 \\
1.12 \\
.94\end{array}$ & & & & & & \\
\hline
\end{tabular}


